\title{
Zeolite-Enhanced Sustainable Pd-Catalyzed C-C Cross-Coupling Reaction: Controlled Release and Capture of Palladium
}

Yanyan Wang,,$^{\dagger}$ Jiaping $\mathrm{Liao}^{\dagger}$ Zunyuan Xie, ${ }^{\dagger}$ Kan Zhang, ${ }^{\dagger}$ Ya Wu, ${ }^{\dagger}$, Ping Zuo, ${ }^{\dagger}$

$$
\text { Weiqiang Zhang, }{ }^{*} \dagger \text { Jiyang Li, } * * \text { and Ziwei Gao*,† }
$$

${ }^{\dagger}$ Key Laboratory of Applied Surface and Colloid Chemistry MOE, School of Chemistry and Chemical Engineering, Shaanxi Normal University, Xi'an 710119, P.R. China. E-mail: zwgao@snnu.edu.cn, zwq@snnu.edu.cn

${ }^{\ddagger}$ State Key Laboratory of Inorganic Synthesis and Preparative Chemistry, College of Chemistry, Jilin University, Changchun, 130012, P.R. China. E-mail: lijiyang@jlu.edu.cn

${ }^{\S}$ College of Chemistry \& Chemical Engineering, Xi'an Shiyou University, Xi'an 710065, P. R. China

\section{Table of Contents}
1. Chemicals
2. Materials Characterization
3. Tables
4. References
5. Figures
6. NMR Spectra 


\section{Chemicals}

Tetra ammine dichloropalladium (II) monohydrate $\left(\mathrm{Pd}\left(\mathrm{NH}_{3}\right)_{4} \mathrm{Cl}_{2} \cdot \mathrm{H}_{2} \mathrm{O}, 97 \%\right.$, Aladdin), tetramethylammonium hydroxide (TMAOH: 25\% in water, ACROS), tetraethylammonium hydroxide (TEAOH, 35\% in water, TCI), tetrapropylammonium hydroxide (TPAOH, 25\% in water, Innochem), tetrabutylammonium hydroxide (TBAOH, 40\% in water, Innochem), phenylacetylene (97\%, Aladdin), 4-iodoanisole (97\%, Aladdin), polyoxyethylene (9-10) octylphenyl ether (Triton X-100, Aladdin).

\section{Materials Characterization}

X-ray powder diffraction (XRD) data were collected on a Bruker D8 Advance diffractometer with $\mathrm{Cu} \mathrm{K} \alpha$ radiation $(\lambda=1.5418 \AA)$. The zeolite morphology was studied by field emission scanning electron microscopy (SEM) Hitachi SU8220 using conventional sample preparation and imaging techniques. ICP analysis was performed on a Thermo Scientific Icap 7600 ICP-OES spectrometer. Thermogravimetric analysis (TGA) was carried out on a TA Q1000 analyzer in $\mathrm{N}_{2}$ with a heating rate of $10{ }^{\circ} \mathrm{C}$ $\min ^{-1}$ from $\mathrm{RT}$ to $800{ }^{\circ} \mathrm{C} . \mathrm{N}_{2}$ adsorptions at $77 \mathrm{~K}$ for the samples were performed on an apparatus of Micromeritics ASAP 2460. Before the adsorption, the samples were pretreated in vacuum at $300{ }^{\circ} \mathrm{C}$ for $6 \mathrm{~h}$ to remove the physical adsorption of water in the holes. Specific surface area was calculated by using the Brunauer-Emmett-Teller (BET) equation. Acidic properties of zeolites were measured by temperature-programmed desorption of ammonia ( $\left.\mathrm{NH}_{3}-\mathrm{TPD}\right)$ method performed on Micromeritics 2920. $0.1 \mathrm{~g}$ samples were purged with pure $\mathrm{He}$ at $350{ }^{\circ} \mathrm{C}$ for $1 \mathrm{~h}$, and then cooled to $80{ }^{\circ} \mathrm{C}$. After being saturated with $\mathrm{NH}_{3}$, the samples were flushed by 
pure $\mathrm{He}$ at $80{ }^{\circ} \mathrm{C}$ for $60 \mathrm{~min}$. Then, the samples were heated to $650{ }^{\circ} \mathrm{C}$ at ramp rate of $10{ }^{\circ} \mathrm{C}$ and the signals were monitored by a thermal conductivity detector (TCD). The transmission electron microscopy (TEM), high-resolution TEM (HRTEM), high-angle annular dark-field scanning transmission electron microscopy (HAADF-STEM), elemental mapping, and Energy Dispersive X-ray (EDX) of a single nanohybrids were carried out on a FEI Tecnai G2 F20 instrument at an accelerating voltage of $200 \mathrm{KV}$. X-ray Photoelectron Spectroscopy (XPS) measurements were performed on an Kratos AXIS ULTRA instrument using a monochromated $\mathrm{Al} \mathrm{K} \alpha \mathrm{X}$-ray source. For high resolution data, the lowest binding energy $\mathrm{C} 1 \mathrm{~s}$ peak was set at $284.6 \mathrm{eV}$ and used as the reference for the other elements. An Agilent $7890 \mathrm{~N}$ gas chromatograph was used for the gas chromatograph (GC) analysis of the samples. The ${ }^{27} \mathrm{Al}$ and ${ }^{29} \mathrm{Si}$ magic angle spinning nuclear magnetic resonance (MAS NMR) spectra were recorded on a Bruker AVANCE III 400 WB spectrometer (Bruker BioSpin AG, Fällanden, Switzerland). The ${ }^{27} \mathrm{Al}$ and ${ }^{29} \mathrm{Si}$ MAS chemical shifts are referenced to the resonances of $\left[\mathrm{Al}\left(\mathrm{H}_{2} \mathrm{O}\right)_{6}\right]^{3+}($ standard $(\mathrm{d}=0.00)$ and 3-(trimethylsilyl)-1-propanesulfonic acid sodium salt (DSS) standard (d=0.0), respectively. ${ }^{1} \mathrm{H}$ and ${ }^{13} \mathrm{C}$ nuclear magnetic resonance (NMR) spectra were recorded on a JEOL JNM-ECZ400S/L1 400 MHz NMR spectrometer. 


\section{Tables}

Table S1. ICP analyses for zeolite USY and the catalyst $0.84 \%$ Pd@USY and 0.084\%Pd@USY. ${ }^{\mathrm{a}}$

\begin{tabular}{lllllll}
\hline Sample & & $\mathrm{Al}$ & $\mathrm{Na}$ & $\mathrm{Pd}$ & $\mathrm{Si}$ & $\mathrm{Pd} /(\mathrm{Si}+\mathrm{Al}) \mathrm{mol} \%$ \\
\hline USY & Exp. (wt \%) & 8.35 & 0.095 & -- & 28.1 & -- \\
& RSD (\%) & 0.1998 & 0.7249 & -- & 0.0062 & -- \\
$0.84 \%$ Pd@USY & Exp. (wt \%) & 8.44 & 0.059 & 0.838 & 28.3 & 0.590 \\
& RSD (\%) & 0.2315 & 1.076 & 0.0754 & 0.2397 & -- \\
& Calcd. (wt \%) & 8.54 & 0.063 & 0.842 & 28.1 & 0.600 \\
$0.084 \%$ Pd@USY & Exp. (wt \%) & 9.04 & 0.067 & 0.084 & 30.3 & 0.056 \\
& RSD (\%) & 0.0212 & 0.1489 & 2.273 & 0.177 & -- \\
& Calcd. (wt \%) & 9.16 & 0.065 & 0.084 & 30.1 & 0.057 \\
\hline
\end{tabular}

${ }^{a}$ Average values of 3 single measurements, general deviation $<\operatorname{RSD}(\%)$. 
Table S2. 0.84\%Pd@USY catalyzed Sonogashira cross-coupling of 4-iodoanisole with phenylacetylene in aqueous solution. ${ }^{\text {a }}$

\begin{tabular}{|c|c|c|c|}
\hline Entry & Base & Time & Yield (\%) \\
\hline 1 & $\mathrm{KOH}$ & $10 \mathrm{~min}$ & $>97$ \\
\hline 2 & $\mathrm{NaOH}$ & $10 \mathrm{~min}$ & $>97$ \\
\hline 3 & TMAOH & $10 \mathrm{~min}$ & $>97$ \\
\hline 4 & TEAOH & $10 \mathrm{~min}$ & $>97$ \\
\hline 5 & ТPAOH & $10 \mathrm{~min}$ & $>97$ \\
\hline 6 & TBAOH & $10 \mathrm{~min}$ & $>97$ \\
\hline $7^{b}$ & TPAOH $\left(\mathrm{Na}^{+}\right)$ & $10 \mathrm{~min}$ & $>97$ \\
\hline 8 & -- & $30 \mathrm{~min}$ & 0 \\
\hline 9 & $\mathrm{TPAOH}^{\mathrm{c}}$ & $3 \mathrm{~h}$ & 0 \\
\hline 10 & $\mathrm{TPAOH}^{\mathrm{d}}$ & $3 \mathrm{~h}$ & 0 \\
\hline
\end{tabular}

${ }^{\text {a }}$ Reaction conditions, unless otherwise stated: 4-iodoanisole $(0.50 \mathrm{mmol})$, phenylacetylene $(0.60$ mmol), catalyst $0.84 \% \mathrm{Pd} @ U S Y(0.01 \mathrm{~g})$, base $(1.0 \mathrm{mmol})$, Triton X-100 $(0.5 \mathrm{~mL})$, in EtOH/ $\mathrm{H}_{2} \mathrm{O}(4$ $\mathrm{mL}, \mathrm{v} / \mathrm{v}=1 / 3)$ at $100{ }^{\circ} \mathrm{C}$, refluxed under aerobic conditions, with yields determined by $\mathrm{GC}$ with the Dodecane as an internal standard, average values of 3-5 single measurements, general deviation $\leq 5 \%$; ${ }^{\mathrm{b}}$ with $3.3 \mathrm{~mol} \% \mathrm{NaCl}$ in the TPAOH;

${ }^{c}$ USY was used as catalyst instead of Pd@USY;

d withoutPd@USY. 
Table S3. ICP analyses of various recycled catalysts that went through the Sonogashira reaction. ${ }^{\text {a }}$

\begin{tabular}{llllll}
\hline Sample $^{\text {b }}$ & $\begin{array}{l}\mathrm{Al} \\
(\mathrm{wt} \%)\end{array}$ & $\begin{array}{l}\mathrm{Pd} \\
(\mathrm{wt} \%)\end{array}$ & $\begin{array}{l}\mathrm{Si} \\
(\mathrm{wt} \%)\end{array}$ & $\begin{array}{l}\mathrm{Si} / \mathrm{Al} \text { ( } /(\mathrm{Si}+\mathrm{Al}) \\
\mathrm{mol} \%\end{array}$ \\
\hline 0.84\%Pd@USY-re1 (NaOH) & 9.18 & 0.45 & 21.7 & 2.3 & 0.38 \\
0.84\%Pd@USY-re2 (TMAOH) & 8.17 & 0.14 & 27.1 & 3.2 & 0.10 \\
0.84\%Pd@USY-re2 (TEAOH) & 8.53 & 0.21 & 28.3 & 3.2 & 0.15 \\
0.84\%Pd@USY-re2 (TPAOH) & 7.65 & 0.754 & 27.3 & 3.4 & 0.56 \\
0.84\%Pd@USY-re2 (TBAOH) & 8.55 & 0.81 & 28.2 & 3.2 & 0.57 \\
0.84\%Pd@USY-re5 (TPAOH) & 7.80 & 0.721 & 26.5 & 3.3 & 0.55 \\
0.84\%Pd@USY-re5 & 7.59 & 0.19 & 25.9 & 3.3 & 0.15 \\
(TPAOH/NaCl) & & & & & \\
0.84\%Pd@USY-re10 (TPAOH) & 7.64 & 0.69 & 25.6 & 3.2 & 0.54 \\
\hline
\end{tabular}

${ }^{a}$ Standard reactant: 4-iodoanisole $(1.50 \mathrm{mmol})$, phenylacetylene $(1.80 \mathrm{mmol})$, catalyst $0.84 \% \mathrm{Pd} @$ USY $(0.03 \mathrm{~g})$, base $(3.0 \mathrm{mmol})$, Triton $\mathrm{X}-100(1.5 \mathrm{~mL})$ in $\mathrm{EtOH} / \mathrm{H}_{2} \mathrm{O}(12 \mathrm{~mL}, \mathrm{v} / \mathrm{v}=1 / 3)$ at $100{ }^{\circ} \mathrm{C}$, refluxed for 20min under aerobic conditions. The experiment and corresponding ICP analyses have been repeated once, with general deviation less than the RSD (\%) values.

${ }^{\mathrm{b}}$ Recovered catalyst of $0.84 \% \mathrm{Pd} @ \mathrm{USY}$ that complete the catalysis reaction in the standard reactant after 1 cycle (0.84\%Pd@USY-re1), 2 cycles (0.84\%Pd@USY-re2), 5 cycles (0.84\%Pd@USY-re5) or 10 cycles (0.84\%Pd@USY-re10), but using NaOH, TMAOH, TEAOH, TPAOH or TBAOH as base.

${ }^{\mathrm{c}}$ Recovered catalyst of $0.84 \% \mathrm{Pd} @ \mathrm{USY}$ that complete the catalysis reaction in the standard reactant but using TPAOH $(3.0 \mathrm{mmol})+\mathrm{NaCl}(0.1 \mathrm{mmol})$ as the base. 
Table S4. The leaching contents of different elements in the reagent by using different bases during the catalytic reaction. ${ }^{\mathrm{a}}$

\begin{tabular}{lll}
\hline Base & $\mathrm{Al} / \mathrm{ppm}$ & $\mathrm{Pd} / \mathrm{ppm}$ \\
\hline TMAOH & 27 & 1.7 \\
TPAOH & 28 & 0.2 \\
TBAOH & 22 & 0.2 \\
\hline
\end{tabular}

a The elements' contents of the solution after catalysis reaction were measured by inductively coupled plasma mass spectrometry (ICP-MS). 
Table S5. Hot filtration test for $0.84 \%$ Pd@ USY catalyzed Sonogashira cross-coupling of 4-iodoanisole with phenylacetylene. ${ }^{\mathrm{a}}$

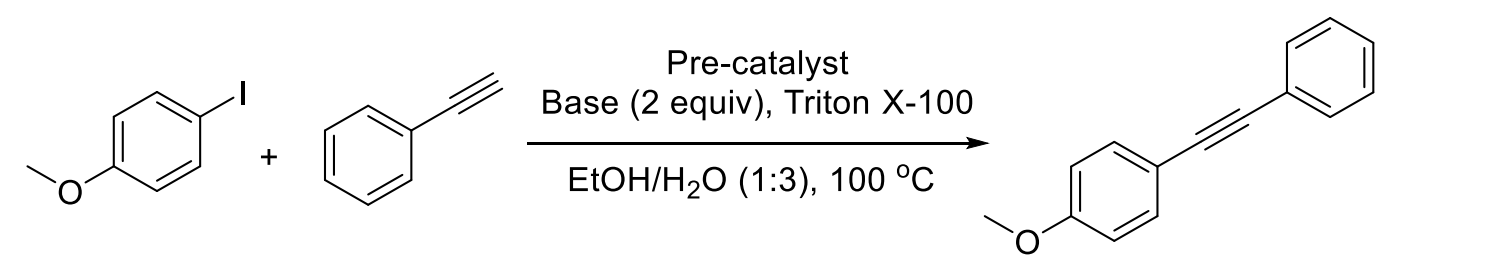

\begin{tabular}{lllll}
\hline Entry & Pre-catalyst & Pd loading $(\mathrm{mol} \mathrm{\% )}$ & Time $(\mathrm{min})$ & Yield $(\%)$ \\
\hline 1 & $0.84 \%$ Pd@USY & 0.158 & 2 & 10 \\
$2^{\mathrm{b}}$ & Clear supernatant from entry 1 & - & 30 & 71 \\
3 & $0.84 \%$ Pd@USY & 0.158 & 2 & 9 \\
$4^{\mathrm{b}}$ & Clear supernatant from entry 3 & - & 30 & 65 \\
5 & $0.84 \%$ Pd@USY & 0.158 & 3 & 17 \\
$6^{\mathrm{b}}$ & Clear supernatant from entry 5 & - & 30 & 92 \\
7 & $\mathrm{Pd}\left(\mathrm{NH}_{3}\right)_{4} \mathrm{Cl}_{2} \cdot \mathrm{H}_{2} \mathrm{O}$ & 0.158 & 10 & $>97$ \\
8 & $\mathrm{Pd}\left(\mathrm{NH}_{3}\right)_{4} \mathrm{Cl}_{2} \cdot \mathrm{H}_{2} \mathrm{O}+\mathrm{USY}$ & 0.158 & 10 & $>97$ \\
9 & $\mathrm{The} \mathrm{recycled} \mathrm{USY} \mathrm{from} \mathrm{entry} 8$ & - & 10 & 95 \\
\hline
\end{tabular}

${ }^{a}$ Reaction conditions, unless otherwise stated: 4-iodoanisole $(1.00 \mathrm{mmol})$, phenylacetylene $(1.20$ mmol), catalyst $(0.158 \mathrm{~mol} \% \mathrm{Pd})$, base $(2.0 \mathrm{mmol})$, Triton $\mathrm{X}-100(0.5 \mathrm{~mL})$, in EtOH/ $\mathrm{H}_{2} \mathrm{O}(1: 3)$ solvent at $100{ }^{\circ} \mathrm{C}$, refluxed under aerobic conditions, with yields determined by GC with the dodecane as an internal standard; ${ }^{\mathrm{b}}$ after two or three minutes of reaction, the supported catalyst was removed by centrifugation at $10000 \mathrm{r}$, and the clear supernatant was further reacted at $100{ }^{\circ} \mathrm{C}$ for $30 \mathrm{~min}$. 
Table S6. Comparison of catalytic performance of 0.84\%Pd@USY with selected previously reported zeolite-supported Pd catalytic systems for the Sonogashira cross-coupling of $p$-iodonanisole with phenyl acetylene.

\begin{tabular}{|c|c|c|c|c|c|c|c|}
\hline Entry & Catalyst [mol\%] & $\begin{array}{l}\text { Reaction } \\
\text { conditions }\end{array}$ & $\begin{array}{l}\mathrm{Pd} \\
{[\mathrm{mol} \%]}\end{array}$ & $\begin{array}{l}\text { Time } \\
{[\mathrm{h}]}\end{array}$ & $\begin{array}{l}\text { Yield } \\
{[\%]}\end{array}$ & TOF & Ref. \\
\hline 1 & $\begin{array}{l}{\left[\mathrm{Pd}\left(\mathrm{NH}_{3}\right)_{4}\right]^{2+} /\left(\mathrm{NH}_{4}\right) \mathrm{Y}} \\
\text { zeolite }\end{array}$ & $\begin{array}{l}\mathrm{DMF} / \mathrm{H}_{2} \mathrm{O}(4 / 1), \\
\mathrm{Et}_{3} \mathrm{~N}, 80^{\circ} \mathrm{C}\end{array}$ & 1 & 3 & 100 & 33.3 & [1] \\
\hline 2 & {$[\mathrm{Pd}] / \mathrm{SBA}-15$} & $\begin{array}{l}\mathrm{DMF} / \mathrm{H}_{2} \mathrm{O}(4 / 1), \\
\mathrm{Et}_{3} \mathrm{~N}, 80^{\circ} \mathrm{C}\end{array}$ & 1 & 6 & 100 & 16.7 & [2] \\
\hline 3 & {$\left[\mathrm{Pd}\left(\mathrm{NH}_{3}\right)_{4}\right] / \mathrm{NaY}$} & $\begin{array}{l}\mathrm{DMF} / \mathrm{H}_{2} \mathrm{O}(4 / 1), \\
\mathrm{Et}_{3} \mathrm{~N}, 80^{\circ} \mathrm{C}\end{array}$ & 1 & 6 & 91 & 15.2 & [2] \\
\hline 4 & {$[\mathrm{Pd}(\mathrm{II})] / \mathrm{NaY}$} & $\begin{array}{l}\mathrm{DMF} / \mathrm{H}_{2} \mathrm{O}(4 / 1), \\
\mathrm{Et}_{3} \mathrm{~N}, 80^{\circ} \mathrm{C}\end{array}$ & 1 & 6 & 50 & 8.3 & [2] \\
\hline 5 & {$[\mathrm{Pd}(0)] / \mathrm{NaY}$} & $\begin{array}{l}\mathrm{DMF} / \mathrm{H}_{2} \mathrm{O}(4 / 1), \\
\mathrm{Et}_{3} \mathrm{~N}, 80^{\circ} \mathrm{C}\end{array}$ & 1 & 6 & 27 & 4.5 & [2] \\
\hline $6^{a}$ & $\mathrm{PdY}$ & $\begin{array}{l}\text { Solvent free, } \\
\mathrm{DBU}, 120^{\circ} \mathrm{C} \\
(300 \mathrm{~W})^{\mathrm{a}}\end{array}$ & 2.35 & $\begin{array}{l}40 \\
\min \end{array}$ & 10 & 6.4 & [3] \\
\hline 7 & AT-Nano CP-Pd ${ }^{0}$ & $\begin{array}{l}\mathrm{H}_{2} \mathrm{O}, \mathrm{K}_{2} \mathrm{CO}_{3} \\
60^{\circ} \mathrm{C}\end{array}$ & 0.06 & 2 & 89 & 741.7 & [4] \\
\hline 8 & $\mathrm{Pd} / \mathrm{SBA}-15$ & $\begin{array}{l}\text { Solvent free, } \\
\text { piperidine, } 75^{\circ} \mathrm{C}\end{array}$ & 0.1 & 1 & 92 & 920 & [5] \\
\hline 9 & $\mathrm{Pd} / \mathrm{SBA}-16$ & $\begin{array}{l}\text { Solvent free, } \\
\text { piperidine, } 80^{\circ} \mathrm{C}\end{array}$ & 0.02 & 2 & 95 & 2375 & [6] \\
\hline $10^{\mathrm{b}}$ & $\begin{array}{l}\mathrm{Pd}^{2+} / \text { mesoporous } \mathrm{NaA} \\
\text { zeolite }\end{array}$ & $\begin{array}{l}\mathrm{DMF} / \mathrm{H}_{2} \mathrm{O} \\
\mathrm{Na}_{2} \mathrm{CO}_{3}, 80^{\circ} \mathrm{C}\end{array}$ & 1.0 & 12 & 91 & 7.6 & [7] \\
\hline $11^{\mathrm{b}}$ & $\begin{array}{l}\mathrm{Pd}^{2+} / \text { mesoporous } \\
\text { sodalite }\end{array}$ & $\begin{array}{l}\mathrm{DMF} / \mathrm{H}_{2} \mathrm{O} \\
\mathrm{Na}_{2} \mathrm{CO}_{3}, 80^{\circ} \mathrm{C}\end{array}$ & 1.0 & 12 & 94 & 7.8 & [7] \\
\hline 12 & 0.84\%Pd@USY & $\begin{array}{l}\mathrm{EtOH} / \mathrm{H}_{2} \mathrm{O}(1 / 3), \\
\mathrm{TPAOH}, 100^{\circ} \mathrm{C}\end{array}$ & $0.158 \%$ & $7 \mathrm{~min}$ & 97 & 5200 & $\begin{array}{l}\text { This } \\
\text { work }\end{array}$ \\
\hline 13 & 0.084\%Pd@USY & $\begin{array}{l}\mathrm{EtOH} / \mathrm{H}_{2} \mathrm{O}(1 / 3), \\
\mathrm{TPAOH}, 100^{\circ} \mathrm{C}\end{array}$ & $0.0032 \%$ & 4 & 97 & 7600 & $\begin{array}{l}\text { This } \\
\text { work }\end{array}$ \\
\hline
\end{tabular}

${ }^{a}$ Microwave-assisted organic synthesis;

${ }^{\mathrm{b}}$ Sonogashira coupling reaction of iodobenzene with phenyl acetylene with conversion. 


\section{Reference}

[1] Djakovitch, L.; Rollet, P., Sonogashira Cross-Coupling Reactions Catalysed by Copper-Free Palladium Zeolites. Adv. Synth. Catal. 2004, 346, 1782-1792.

[2] Rollet, P.; Kleist, W.; Dufaud, V.; Djakovitch, L., Copper-Free Heterogeneous Catalysts for the Sonogashira Cross-Coupling Reaction: Preparation, Characterisation, Activity and Applications for Organic Synthesis. J. Mol. Catal. A: Chem. 2005, 241, 39-51.

[3] Chang, W.; Shin, J.; Chae, G.; Jang, S. R.; Ahn, B. J., Microwave-Assisted Sonogashira Cross-Coupling Reaction Catalyzed by Pd-MCM-41 under Solvent-Free Conditions. J. Ind. Eng. Chem. 2013, 19, 739-743.

[4] Baghbanian, S. M.; Yadollahy, H.; Tajbakhsh, M.; Farhang, M.; Biparva, P., Palladium Nanoparticles Supported on Natural Nanozeolite Clinoptilolite as a Catalyst for Ligand and Copper-Free C-C and C-O Cross Coupling Reactions in Aqueous Medium. RSC Adv. 2014, 4, $62532-62543$.

[5] Sarkar, S. M.; Rahman, M. L.; Yusoff, M. M., Highly Active Thiol-Functionalized SBA-15 Supported Palladium Catalyst for Sonogashira and Suzuki-Miyaura Cross-Coupling Reactions. RSC Adv. 2015, 5, 1295-1300.

[6] Sarkar, S. M.; Rahman, M. L.; Yusoff, M. M., Heck, Suzuki and Sonogashira Cross-Coupling Reactions Using Ppm Level of SBA-16 Supported Pd-Complex. New J. Chem. 2015, 39, 3564-3570.

[7] Choi, M.; Lee, D.-H.; Na, K.; Yu, B.-W.; Ryoo, R., High Catalytic Activity of Palladium(II)-Exchanged Mesoporous Sodalite and NaA Zeolite for Bulky Aryl Coupling Reactions: Reusability under Aerobic Conditions. Angew. Chem. Int. Ed. 2009, 48, 3673-3676. 


\section{Figures}

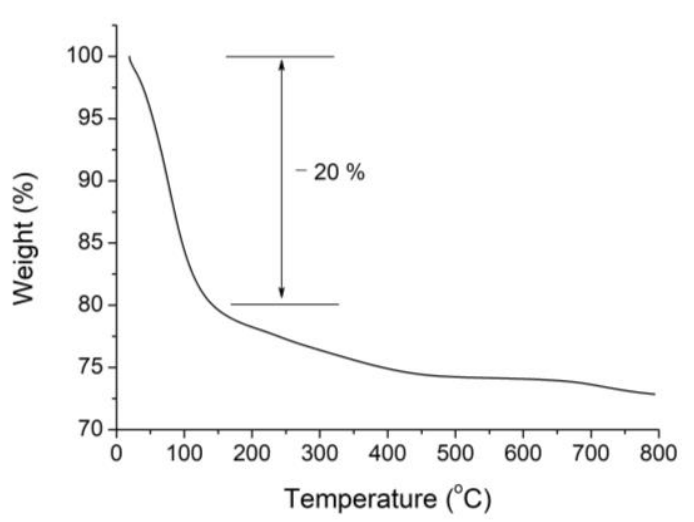

(a)

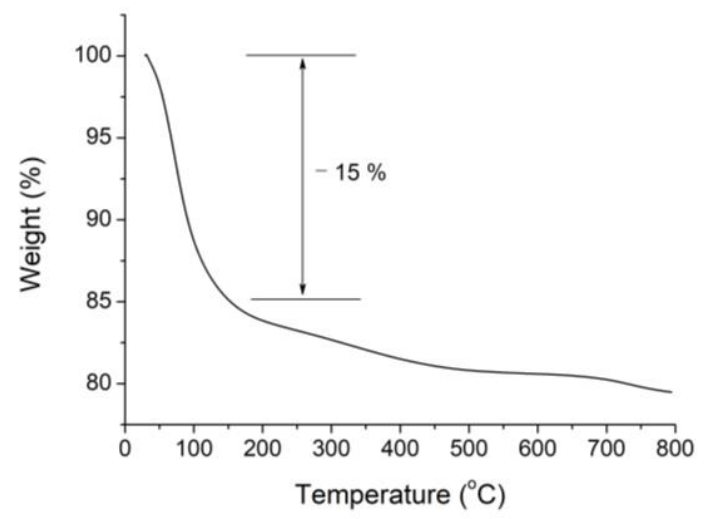

(c)

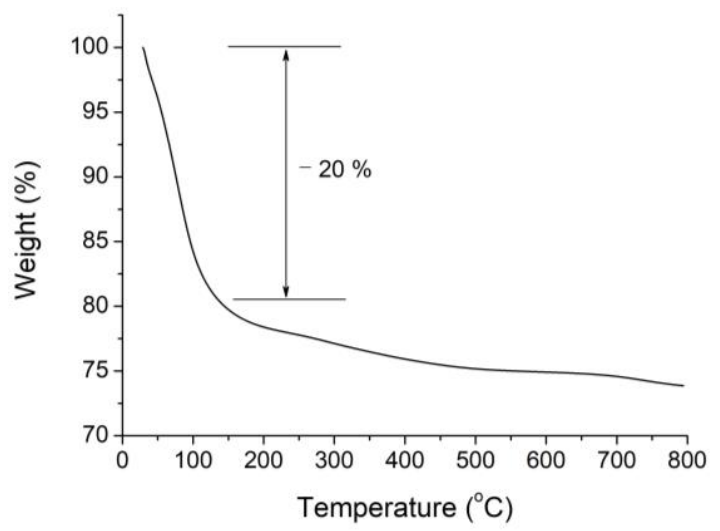

(b)

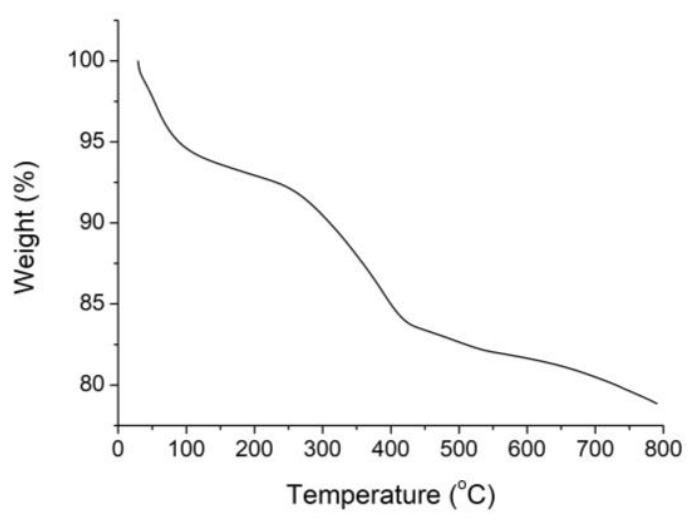

(d)

Figure S1. TGA curves of (a) USY, (b) 0.84\%Pd@USY, (c) 0.084\%Pd@USY and (d) 0.84\%Pd@USY-re1, respectively. It shows weight loss of 20\%, 20\% and 15\% around $100{ }^{\circ} \mathrm{C}$ for USY, 0.84\%Pd@USY and 0.084\%Pd@USY, respectively, which is attributed to the loss of physical adsorption of water molecules. The weight loss around $300{ }^{\circ} \mathrm{C}$ for $0.84 \% \mathrm{Pd} @$ USY-re1 indicates the organic residue in the channel. 

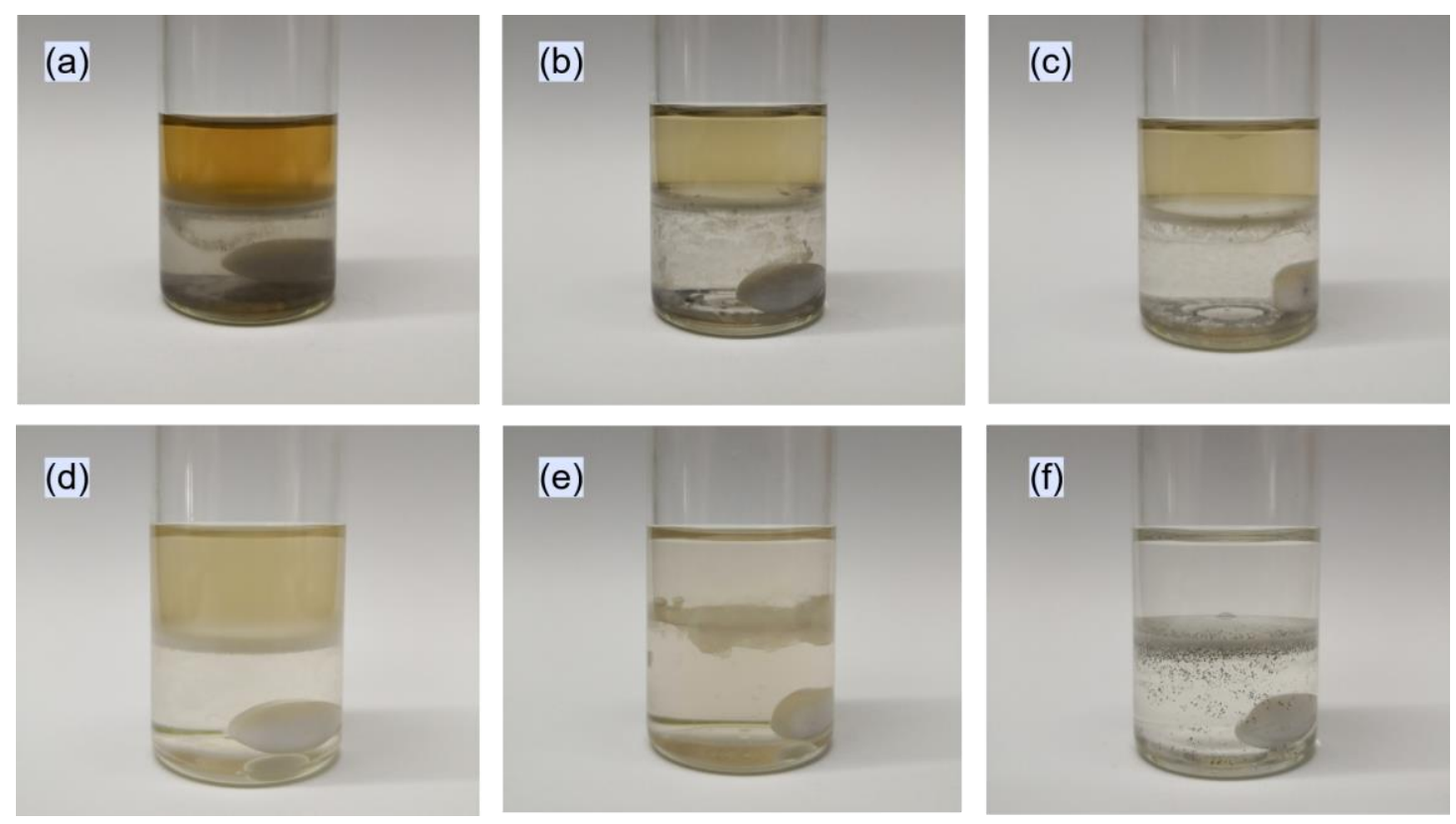

Figure S2. Representative photographs of the reagent after being catalyzed by 0.84\%Pd@USY. Base used: (a) NaOH; (b) TMAOH; (c) TEAOH; (d) TPAOH; (e) $\mathrm{TBAOH}$; (f) TPAOH with trace $\mathrm{NaCl}$ as additive.

In order to observe the catalyst more clearly, $4-5 \mathrm{~mL}$ of dichloromethane was added to the reagent after the catalytic reaction was completed. Obviously, 0.84\%Pd@USY materials are located at the junction of the two layers. In (a), (b), (c) and (f), there are some black particles dispersing in the dichloromethane layer. But no obvious black particles in (d) and (e).

Reaction conditions: bromobenzene $(0.5 \mathrm{mmol})$, 4-Methoxyphenylboronic acid $(0.6$ mmol), 0.84\%Pd@USY $(0.01 \mathrm{~g})$, base $(1 \mathrm{mmol}), \mathrm{H}_{2} \mathrm{O}(4 \mathrm{~mL})$, Triton $(0.5 \mathrm{~mL}), 100{ }^{\circ} \mathrm{C}$, $20 \mathrm{~min}$ without gas protection. 
(a)

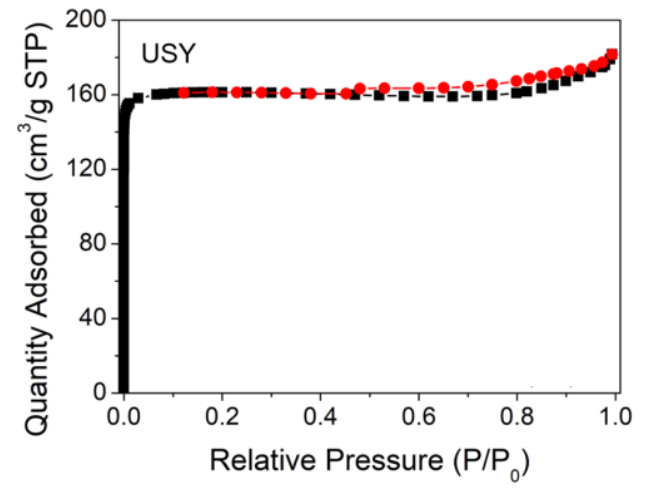

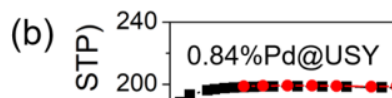

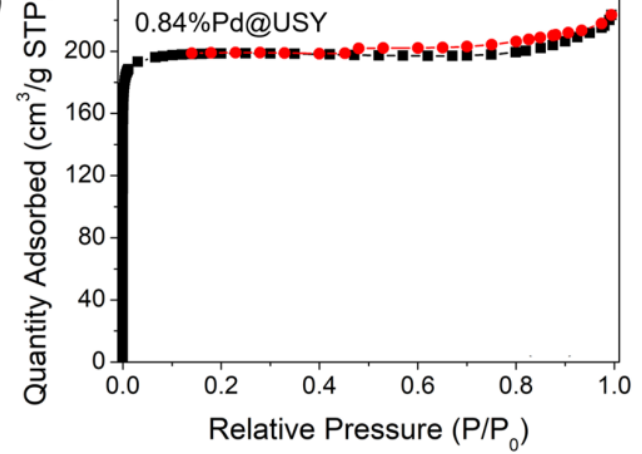

Figure S3. $\mathrm{N}_{2}$ adsorption/desorption isotherms of (a) USY and (b) $0.84 \% \mathrm{Pd} @$ USY.

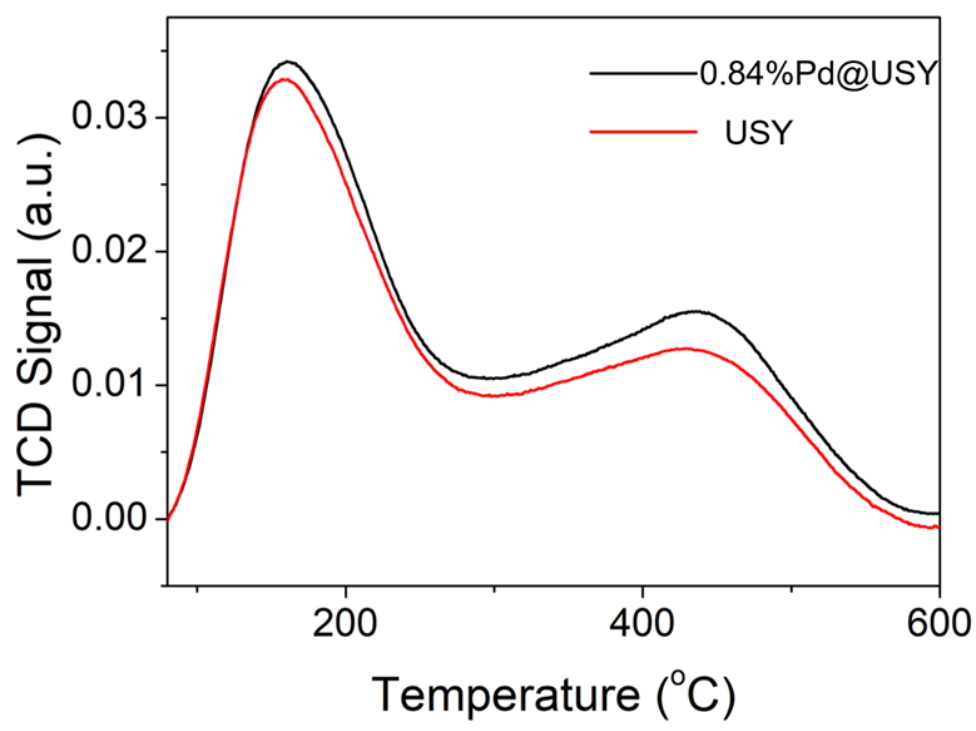

Figure S4. $\mathrm{NH}_{3}$-TPD profiles of USY and 0.84\%Pd@USY. 

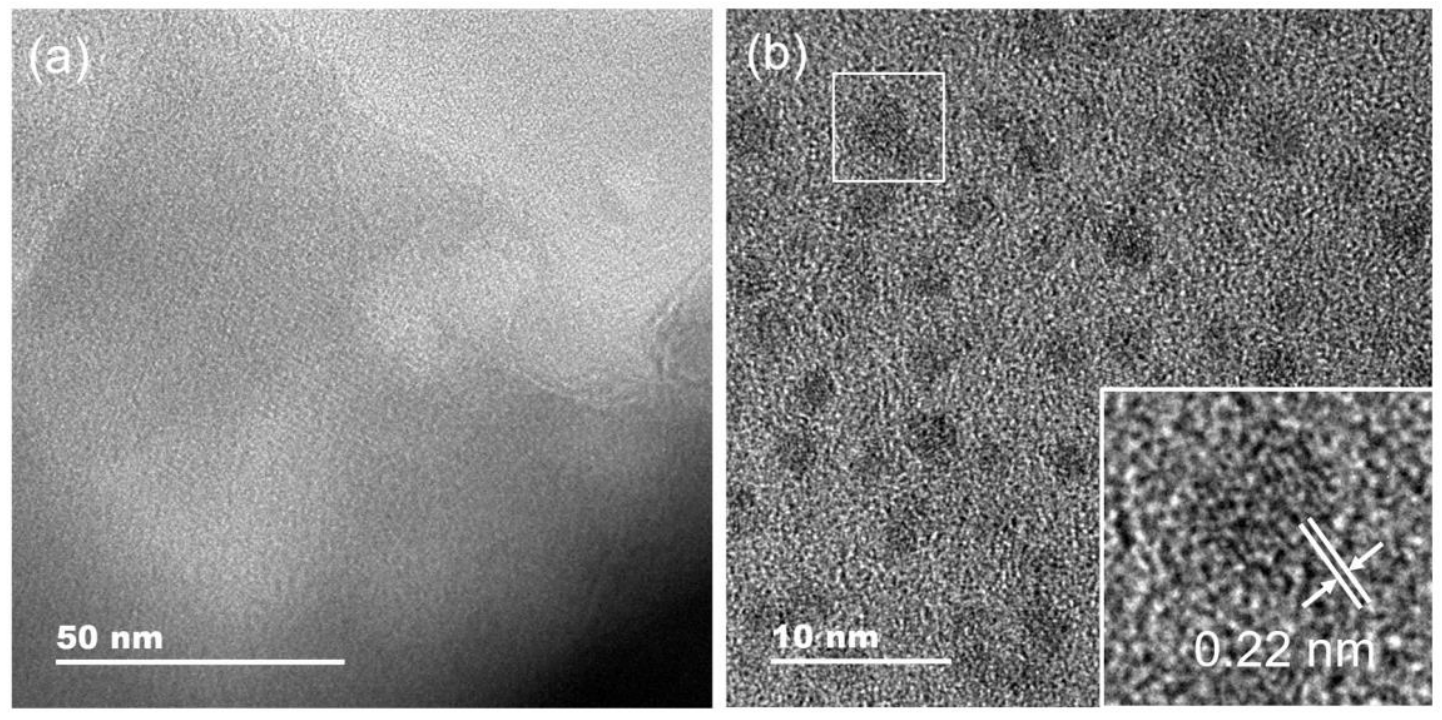

Figure S5. (a) TEM image of $0.84 \% \mathrm{Pd@USY-re10} \mathrm{at} \mathrm{high} \mathrm{magnification,} \mathrm{showing}$ that no nanoparticle of Pd observed on the zeolite; (b) TEM image obtained after irradiating the $0.84 \% \mathrm{Pd} @ U S Y-r e 10$ in (a) under high-resolution TEM (HRTEM) electron beam for several minutes, showing the emerging of Pd nanoparticles on the zeolite. It indicates that Pd species are highly dispersed on the recycled supported catalyst. 


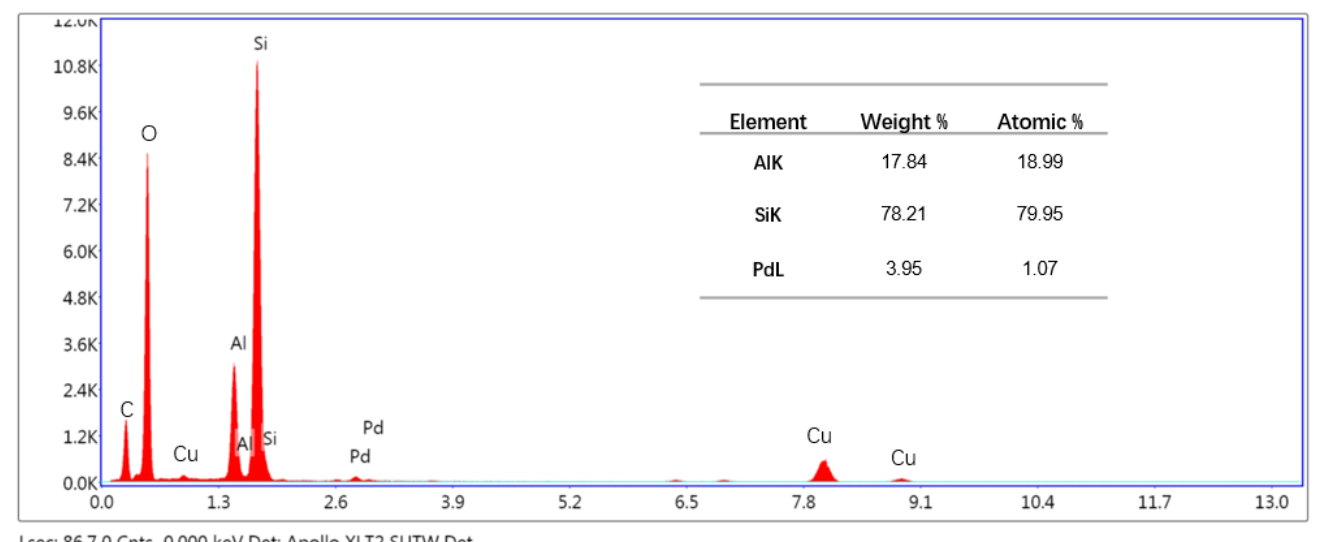

(a)

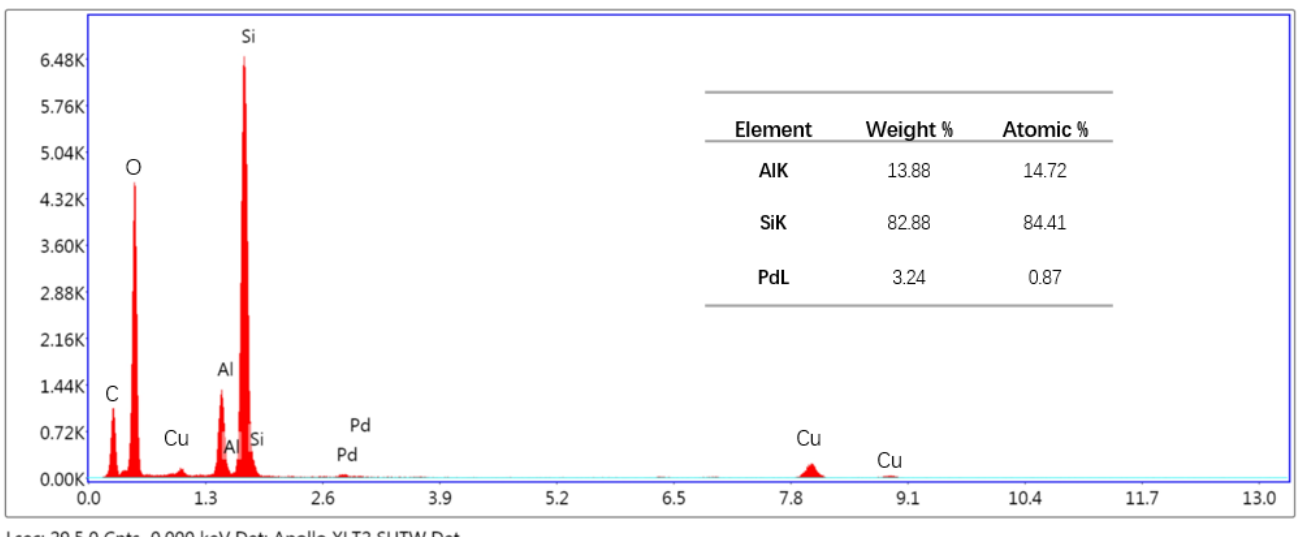

(b)

Figure S6. energy dispersive spectrometer (EDS) analyses of (a) 0.84\%Pd@USY and (b) 0.84\%Pd@USY-re10. It indicates the as-synthesized 0.84\%Pd@USY and used $0.84 \% \mathrm{Pd} @$ USY-re10 are composed of only Al, Si, Pd and O elements, whereas the signals for $\mathrm{Cu}$ and $\mathrm{C}$ are from the substrate. 


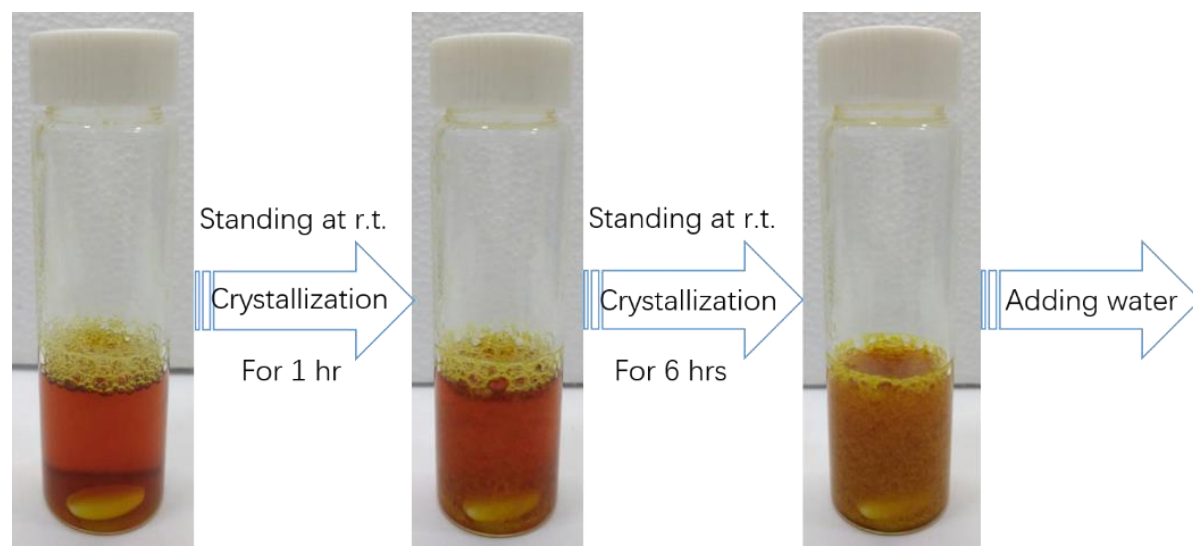

Figure S7. The pictures for the crystallization process of product 1-methoxy-4-(phenylethynyl)benzene in reaction agent.

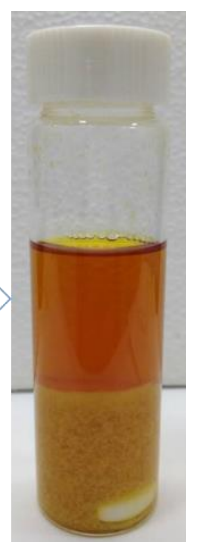

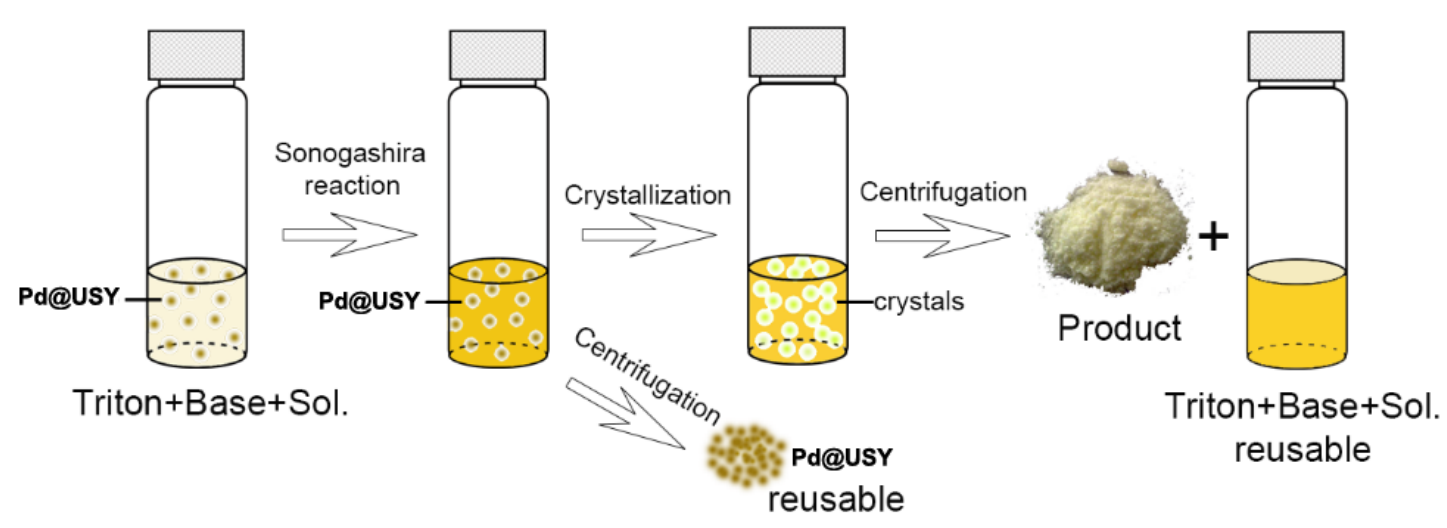

Figure S8. Scheme for recycling and reusing the catalyst and reactant. 


\section{Characterization of Compounds}

\section{1-methoxy-4-(phenylethynyl)benzene}

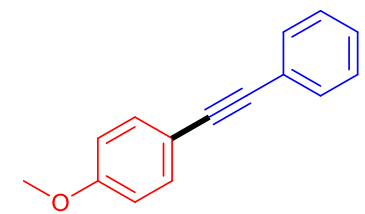

Pale yellow solid, yield $202 \mathrm{mg}(97 \%) .{ }^{1} \mathrm{H}$ NMR $\delta\left(400 \mathrm{MHz}, \mathrm{CDCl}_{3}\right) 7.55-7.44(4 \mathrm{H}, \mathrm{m}), 7.38-$ $7.28(3 \mathrm{H}, \mathrm{m}), 6.91-6.86(2 \mathrm{H}, \mathrm{m}), 3.83(3 \mathrm{H}, \mathrm{s}) ;{ }^{13} \mathrm{C} \mathrm{NMR} \delta\left(101 \mathrm{MHz}, \mathrm{CDCl}_{3}\right)$ 159.69, 133.14, $131.56,131.55,131.54,131.52,128.42,128.41,128.40,128.38,128.02,123.67,115.45,114.08$, $89.44,88.14,55.39$.

\section{1,2-diphenylethyne}

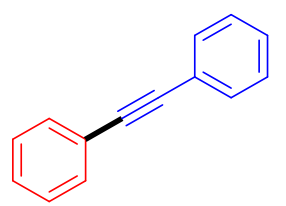

Pale yellow solid, yield $176 \mathrm{mg}(99 \%) .{ }^{1} \mathrm{H}$ NMR $\delta\left(400 \mathrm{MHz}, \mathrm{CDCl}_{3}\right) 7.66-7.51(4 \mathrm{H}, \mathrm{m}), 7.37$ $(6 \mathrm{H}, \mathrm{dt}, J 3.9,1.5) ;{ }^{13} \mathrm{C}$ NMR $\delta\left(101 \mathrm{MHz}, \mathrm{CDCl}_{3}\right) 131.77,131.76,128.51,128.42,123.39$, 89.54.

\section{1-methyl-4-(phenylethynyl)benzene}

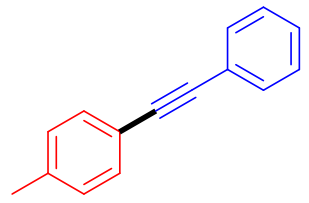

Pale yellow solid, yield $190 \mathrm{mg}(99 \%) .{ }^{1} \mathrm{H}$ NMR $\delta\left(400 \mathrm{MHz}, \mathrm{CDCl}_{3}\right) 7.57-7.50(2 \mathrm{H}, \mathrm{m}), 7.47-$ $7.40(2 \mathrm{H}, \mathrm{m}), 7.39-7.28(3 \mathrm{H}, \mathrm{m}), 7.22-7.12(2 \mathrm{H}, \mathrm{m}), 2.38(3 \mathrm{H}, \mathrm{s}) ;{ }^{13} \mathrm{C}$ NMR $\delta(101 \mathrm{MHz}$, $\left.\mathrm{CDCl}_{3}\right) 138.48,131.69,131.68,131.67,131.66,131.65,131.64,131.62,131.59,131.56,131.55$, $131.54,131.54,129.24,129.23,129.22,129.21,129.19,129.18,129.17,128.44,128.43,128.42$, $128.41,128.39,128.38,128.37,128.16,123.55,120.26,89.64,88.80,21.61$.

\section{1-nitro-3-(phenylethynyl)benzene}<smiles>O=[N+]([O-])c1cccc(C#Cc2ccccc2)c1</smiles>

Pale yellow solid, yield $221 \mathrm{mg}(99 \%) .{ }^{1} \mathrm{H}$ NMR $\delta\left(400 \mathrm{MHz}, \mathrm{CDCl}_{3}\right) 8.41-8.33(1 \mathrm{H}, \mathrm{m}), 8.16$ $(1 \mathrm{H}$, ddd, $J$ 8.3, 2.3, 1.1), $7.83-7.78(1 \mathrm{H}, \mathrm{m}), 7.57-7.49(3 \mathrm{H}, \mathrm{m}), 7.38(3 \mathrm{H}, \mathrm{dd}, J$ 5.0, 1.8); ${ }^{13} \mathrm{C}$ NMR $\delta\left(101 \mathrm{MHz}, \mathrm{CDCl}_{3}\right) 148.18,137.34,131.89,129.48,129.18,128.63,126.46,125.20$, $122.98,122.26,92.03,87.00$. 


\section{1,4-bis(phenylethynyl)benzene}

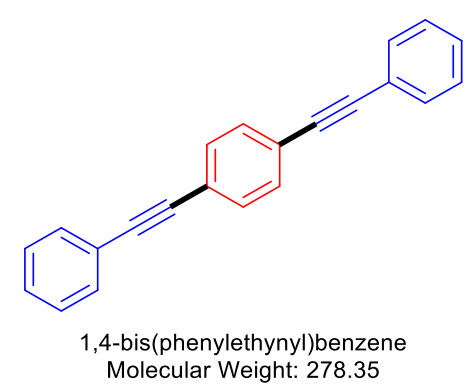

Yellow solid, yield $276 \mathrm{mg}(99 \%) .{ }^{1} \mathrm{H}$ NMR $\delta\left(400 \mathrm{MHz}, \mathrm{CDCl}_{3}\right) 7.56(4 \mathrm{H}, \mathrm{dd}, J$ 6.5, 3.3), 7.53 (4 H, s), 7.37 (6 H, dd, J 5.0, 1.9); ${ }^{13} \mathrm{C}$ NMR $\delta\left(101 \mathrm{MHz}, \mathrm{CDCl}_{3}\right)$ 131.74, 131.65, 128.59, 128.51, $123.19,123.13,91.35,89.23$.

\section{4-methoxy-1,1'-biphenyl}<smiles>COc1ccc(-c2ccccc2)cc1</smiles>

Gray solid, yield $183 \mathrm{mg}(99 \%) .{ }^{1} \mathrm{H}$ NMR $\delta\left(400 \mathrm{MHz}, \mathrm{CDCl}_{3}\right) 7.65-7.53(4 \mathrm{H}, \mathrm{m}), 7.45(2 \mathrm{H}$, $\mathrm{dd}, J 11.0,4.1), 7.35(1 \mathrm{H}, \mathrm{d}, J 7.5), 7.08-6.96(2 \mathrm{H}, \mathrm{m}), 3.88(3 \mathrm{H}, \mathrm{s}) ;{ }^{13} \mathrm{C}$ NMR $\delta(101 \mathrm{MHz}$, $\left.\mathrm{CDCl}_{3}\right)$ 159.25, 140.94, 133.87, 128.85, 128.28, 128.26, 126.86, 126.84, 126.78, 114.31, 55.45.

\section{1-(4-methoxyphenyl)naphthalene}<smiles>COc1ccc(-c2cccc3ccccc23)cc1</smiles>

Slivery-white solid, yield $232 \mathrm{mg}(99 \%) .{ }^{1} \mathrm{H}$ NMR $\delta\left(400 \mathrm{MHz}, \mathrm{CDCl}_{3}\right) 8.05-7.82(3 \mathrm{H}, \mathrm{m}), 7.58$ - 7.43 (6 H, m), 7.08 (2 H, t, J 6.1), 3.93 (3 H, s); ${ }^{13} \mathrm{C} \mathrm{NMR} \delta$ (101 MHz, $\mathrm{CDCl}_{3}$ ) 159.03, 140.00, $133.93,133.21,131.91,131.24,128.38,127.45,127.04,126.18,126.05,125.83,125.54,113.83$, $113.81,55.47$.

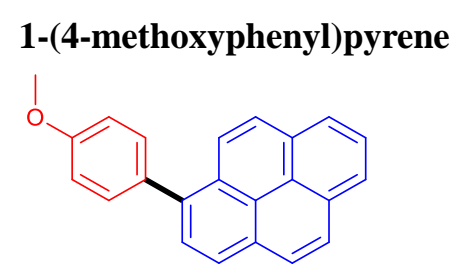

Pale yellow solid, yield $305 \mathrm{mg}(99 \%) .{ }^{1} \mathrm{H}$ NMR $\delta\left(400 \mathrm{MHz}, \mathrm{CDCl}_{3}\right) 8.26-7.95(9 \mathrm{H}, \mathrm{m}), 7.67-$ $7.51(2 \mathrm{H}, \mathrm{m}), 7.12\left(2 \mathrm{H}, \mathrm{d}, J\right.$ 8.7), $3.94(3 \mathrm{H}, \mathrm{s}) ;{ }^{13} \mathrm{C} \mathrm{NMR} \delta\left(101 \mathrm{MHz}, \mathrm{CDCl}_{3}\right) 159.10,137.57$, 133.66, 131.76, 131.62, 131.12, 130.47, 128.69, 127.81, 127.57, 127.55, 127.45, 127.43, 127.37, $126.08,125.48,125.12,125.06,124.84,124.77,113.97,55.51$. 


\section{4,4'-dimethoxy-1,1'-biphenyl}

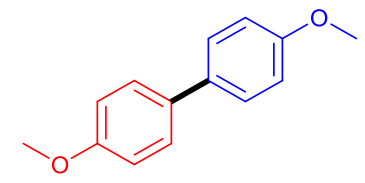

Slivery-white solid, yield $214 \mathrm{mg}(100 \%) .{ }^{1} \mathrm{H}$ NMR $\delta\left(400 \mathrm{MHz}, \mathrm{CDCl}_{3}\right) 7.57-7.41(4 \mathrm{H}, \mathrm{m})$, $7.04-6.91(4 \mathrm{H}, \mathrm{m}), 3.85(6 \mathrm{H}, \mathrm{s}) ;{ }^{13} \mathrm{C} \mathrm{NMR} \delta\left(101 \mathrm{MHz}, \mathrm{CDCl}_{3}\right) 158.77,133.56,127.83,114.25$, 55.43 . 
6. NMR Spectra

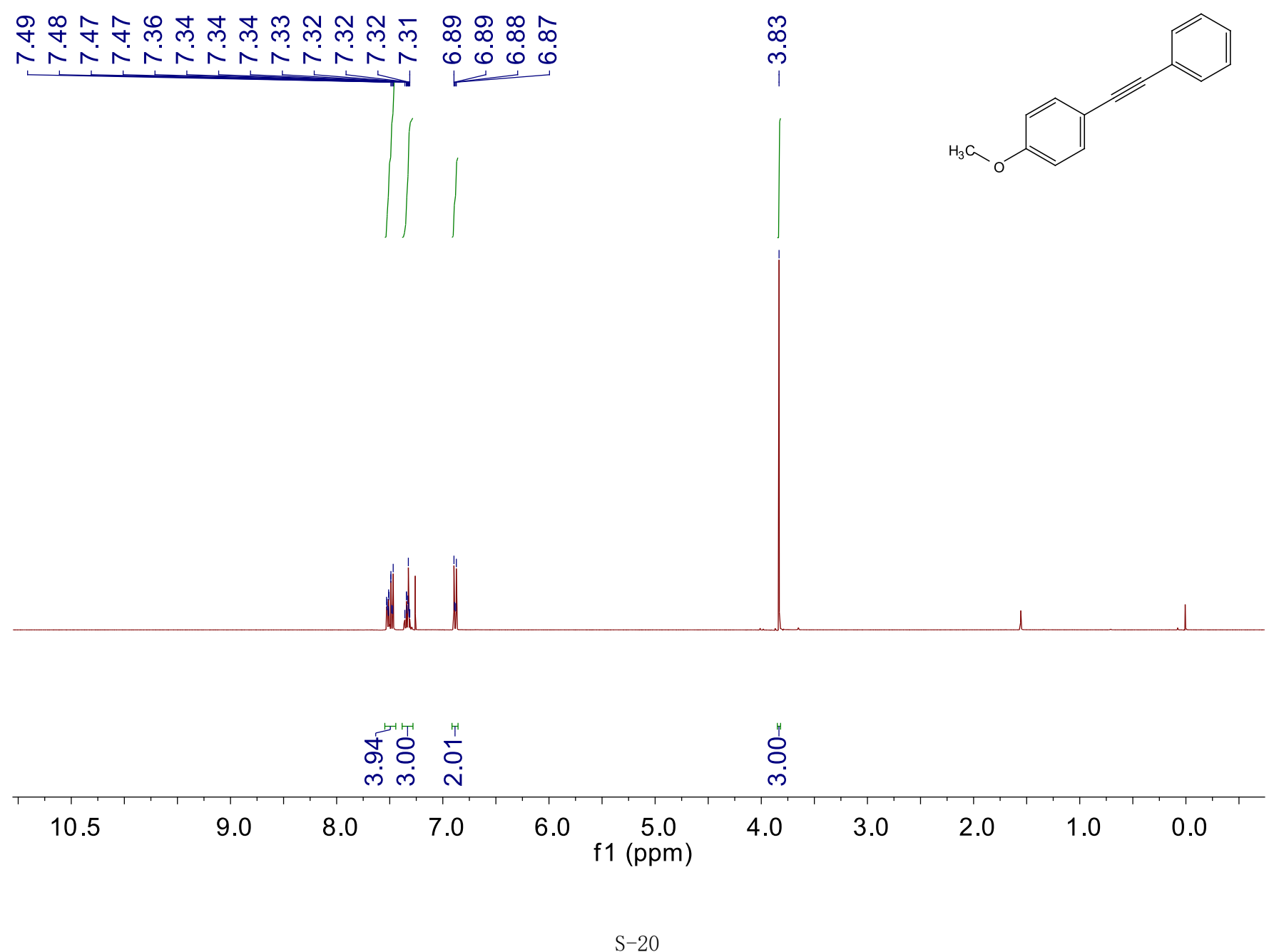



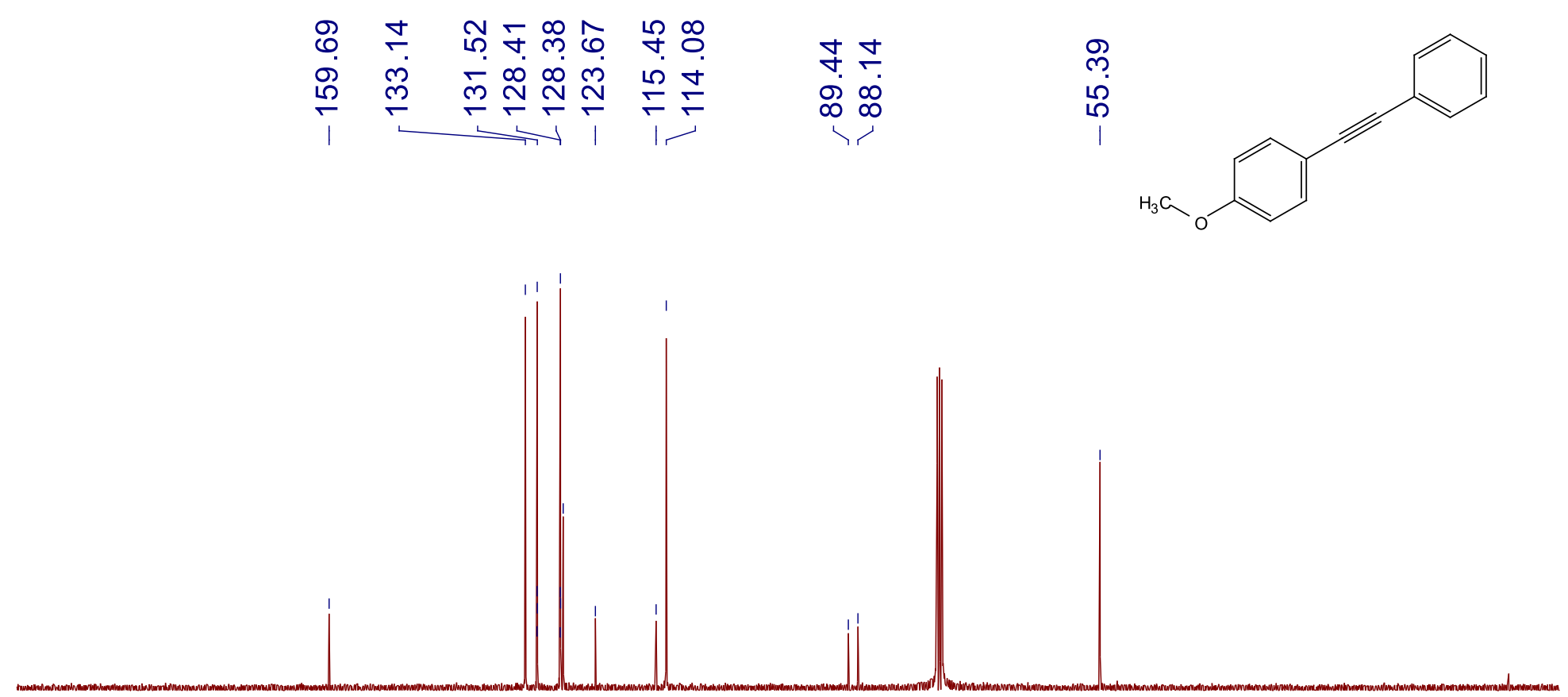

\begin{tabular}{|c|c|c|c|c|c|c|c|c|c|}
\hline 200 & 180 & 160 & 140 & 120 & $\begin{array}{c}100 \\
\mathrm{f} 1(\mathrm{ppm})\end{array}$ & 80 & 60 & 40 & 20 \\
\hline
\end{tabular}




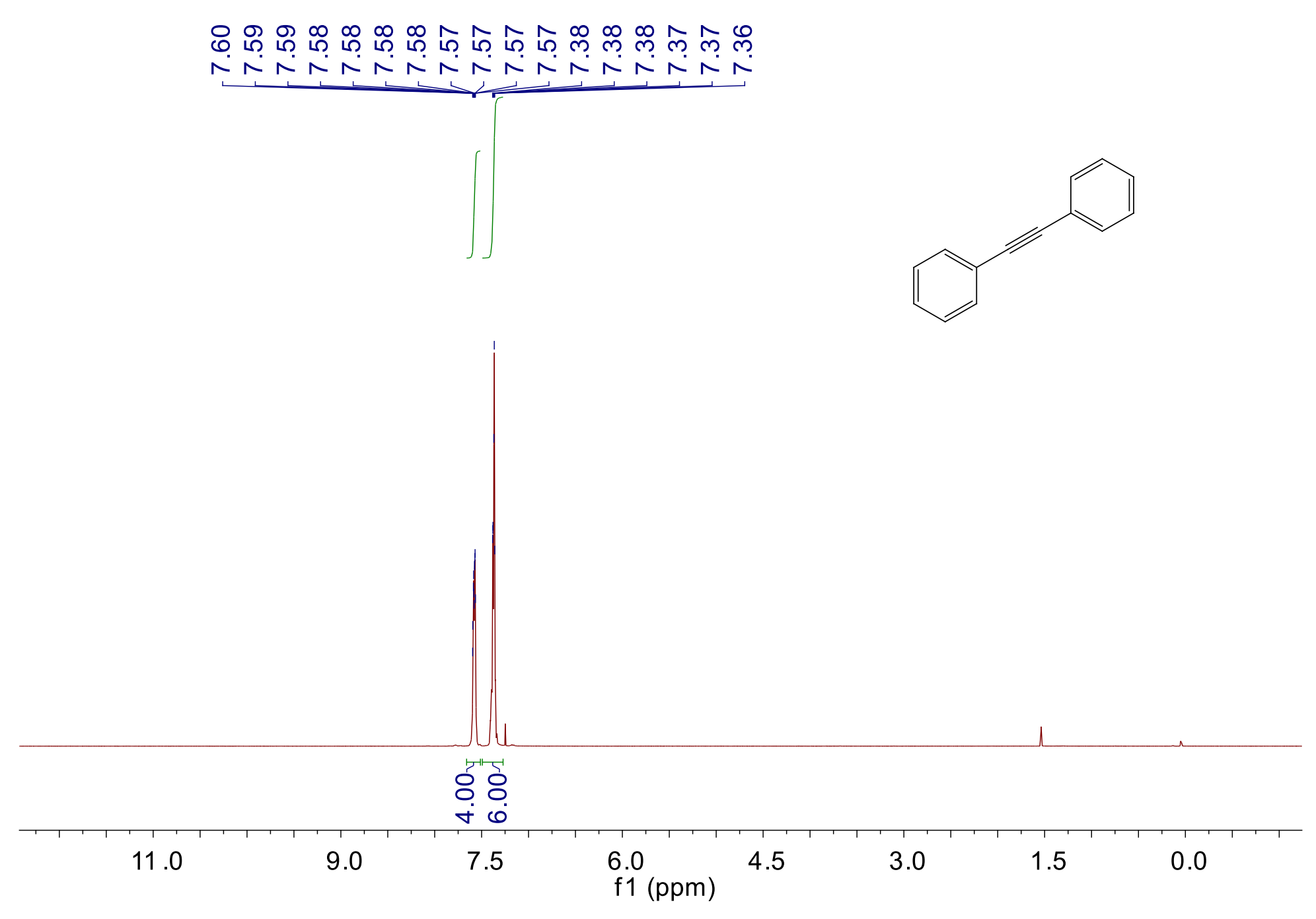




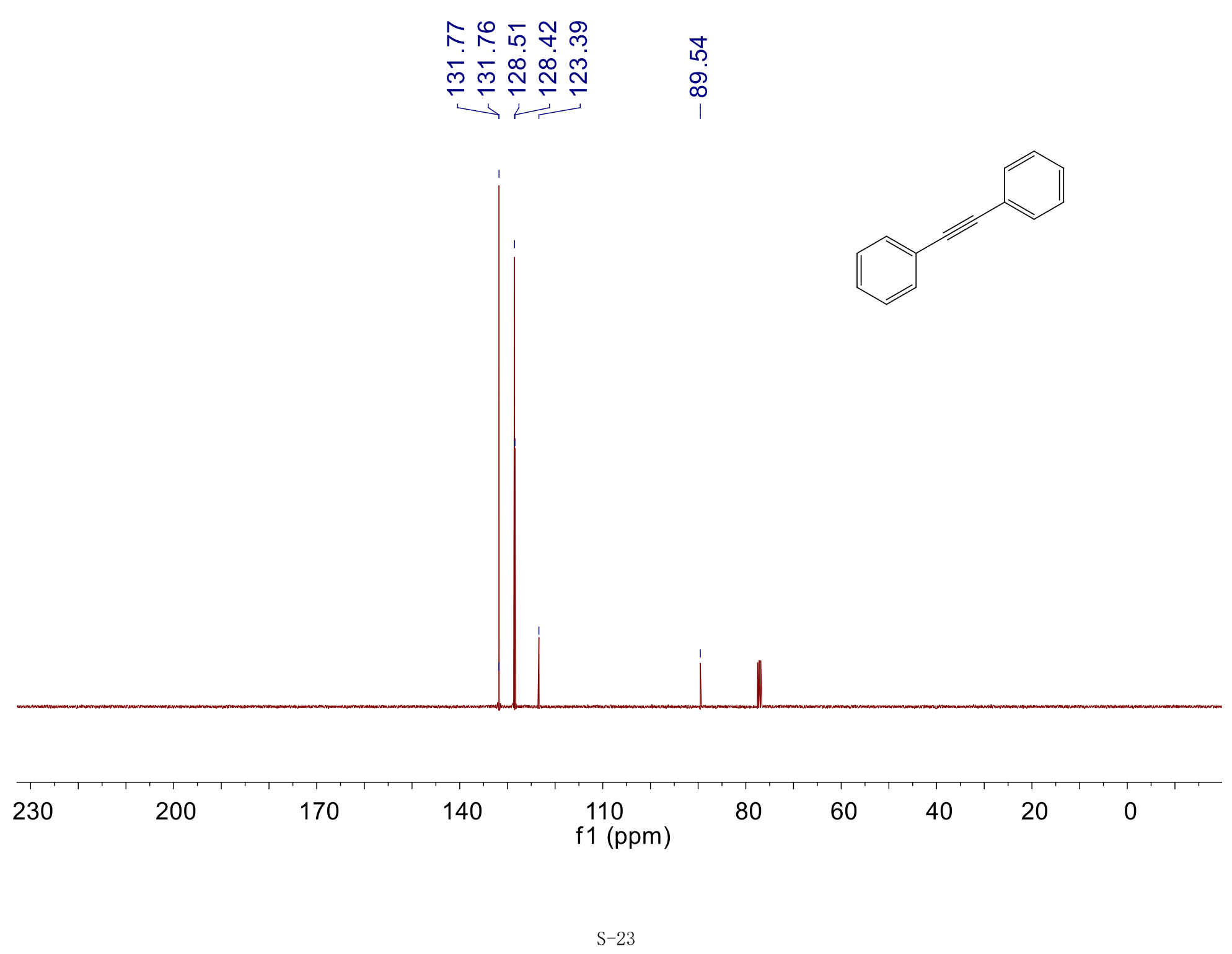




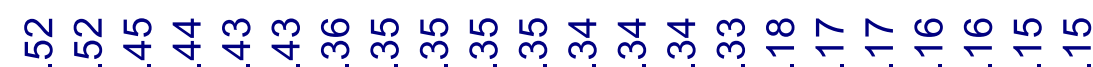

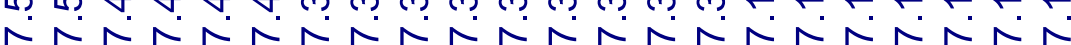

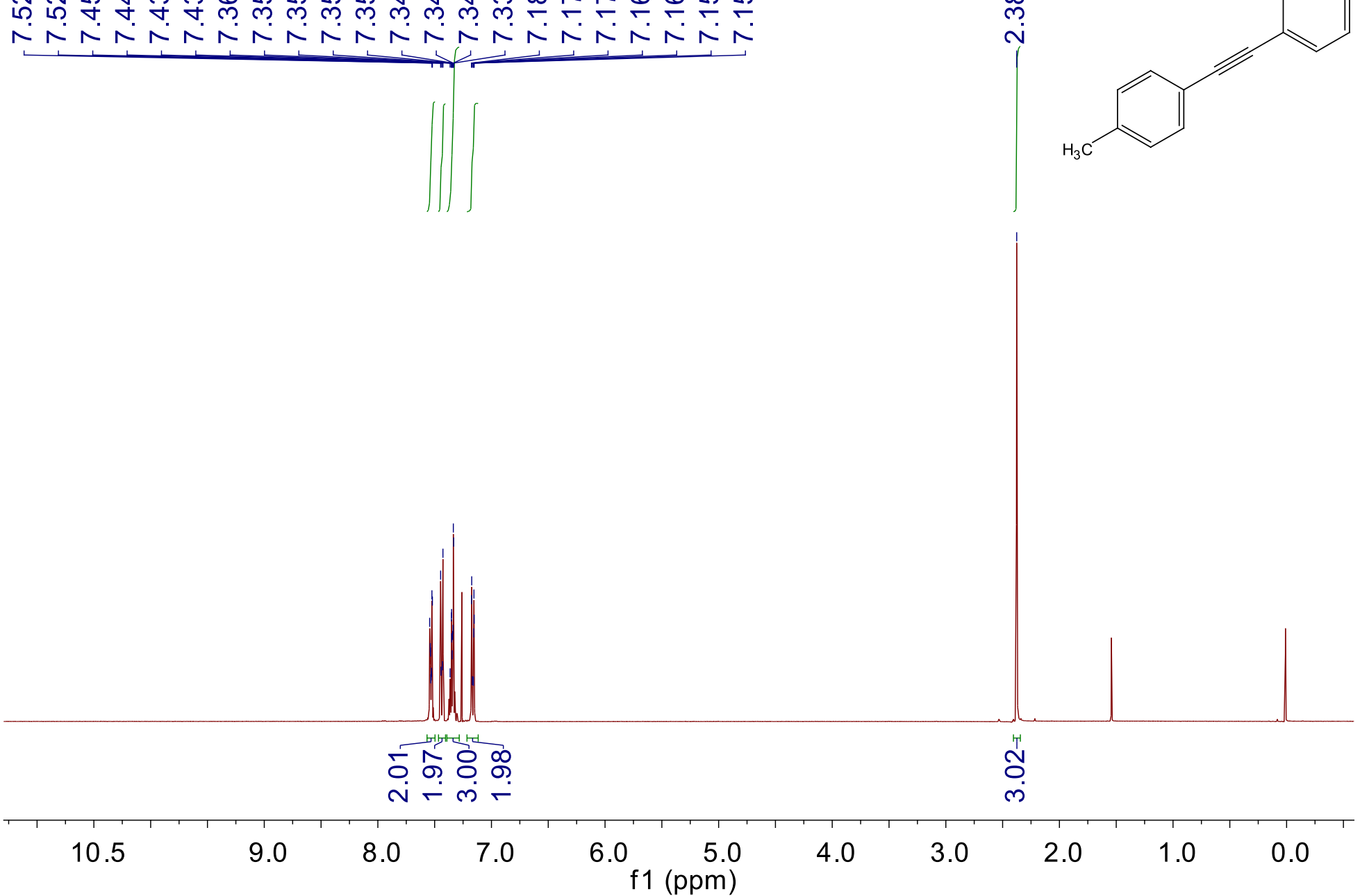




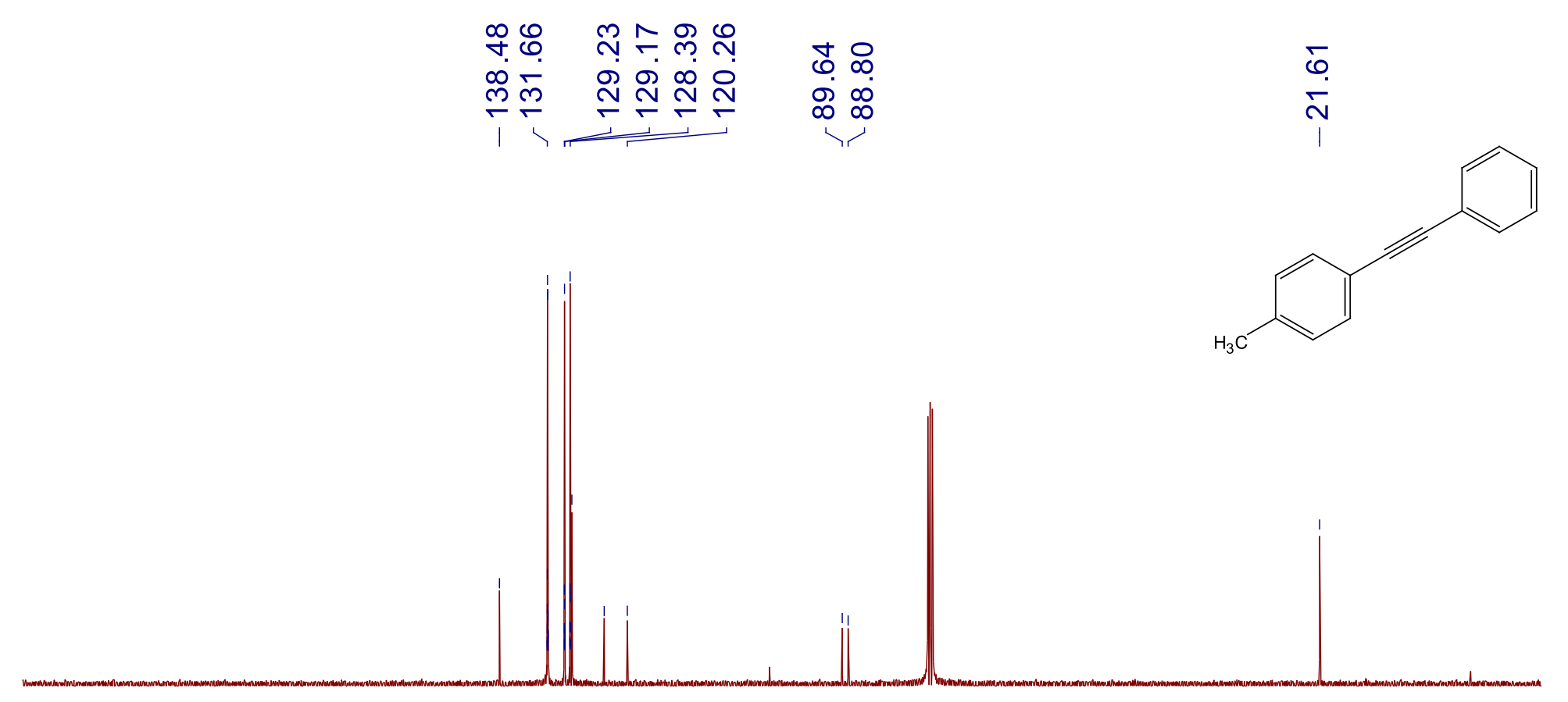

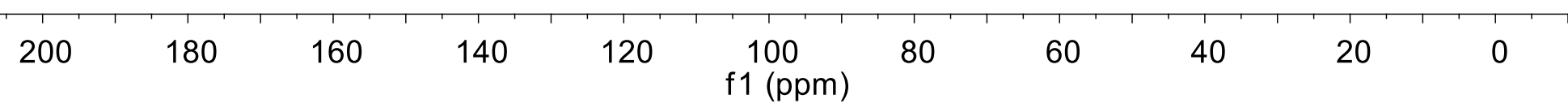


అִ

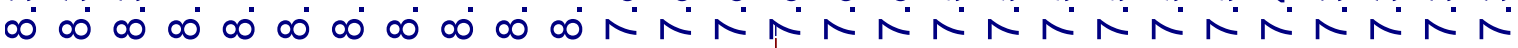

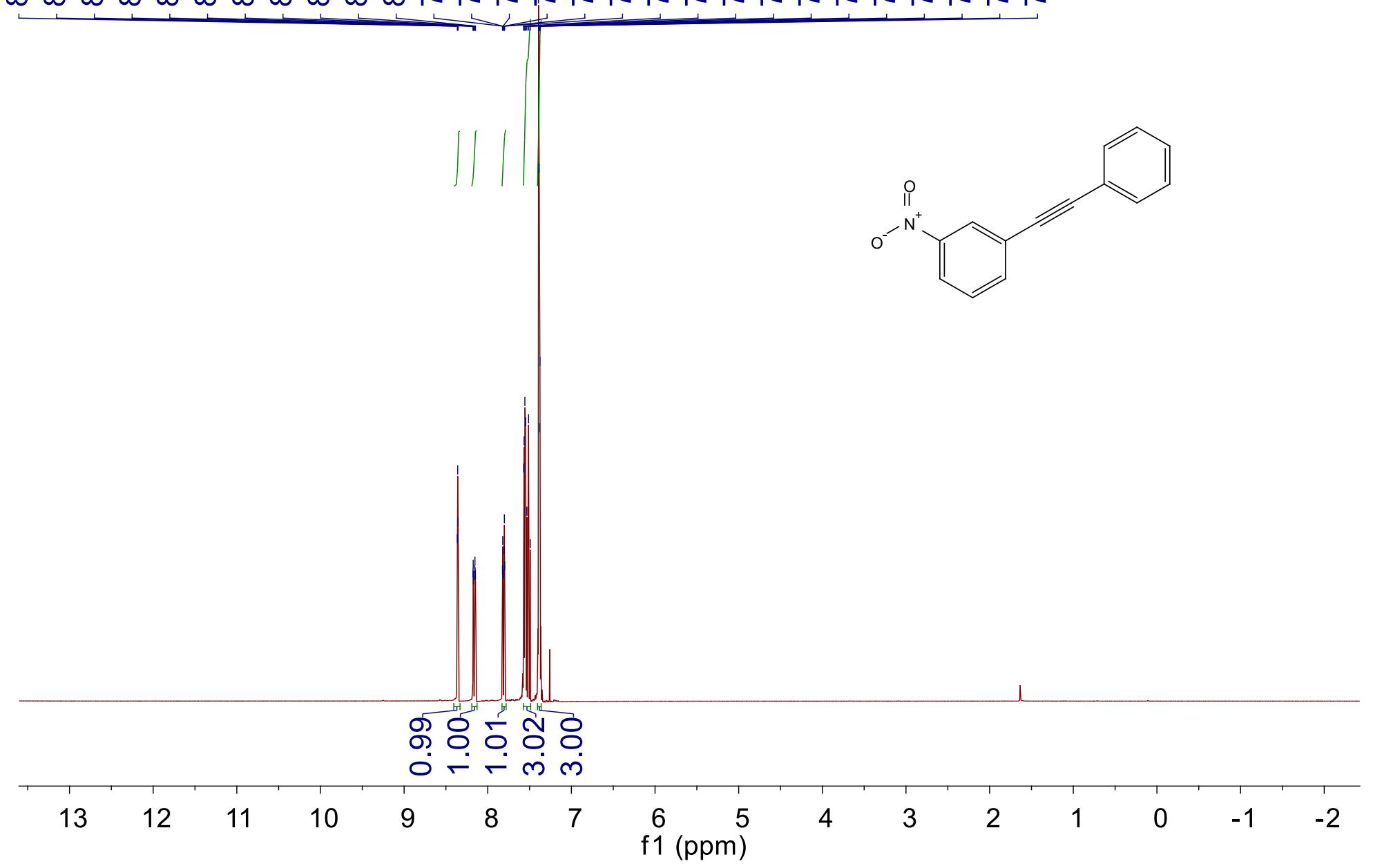



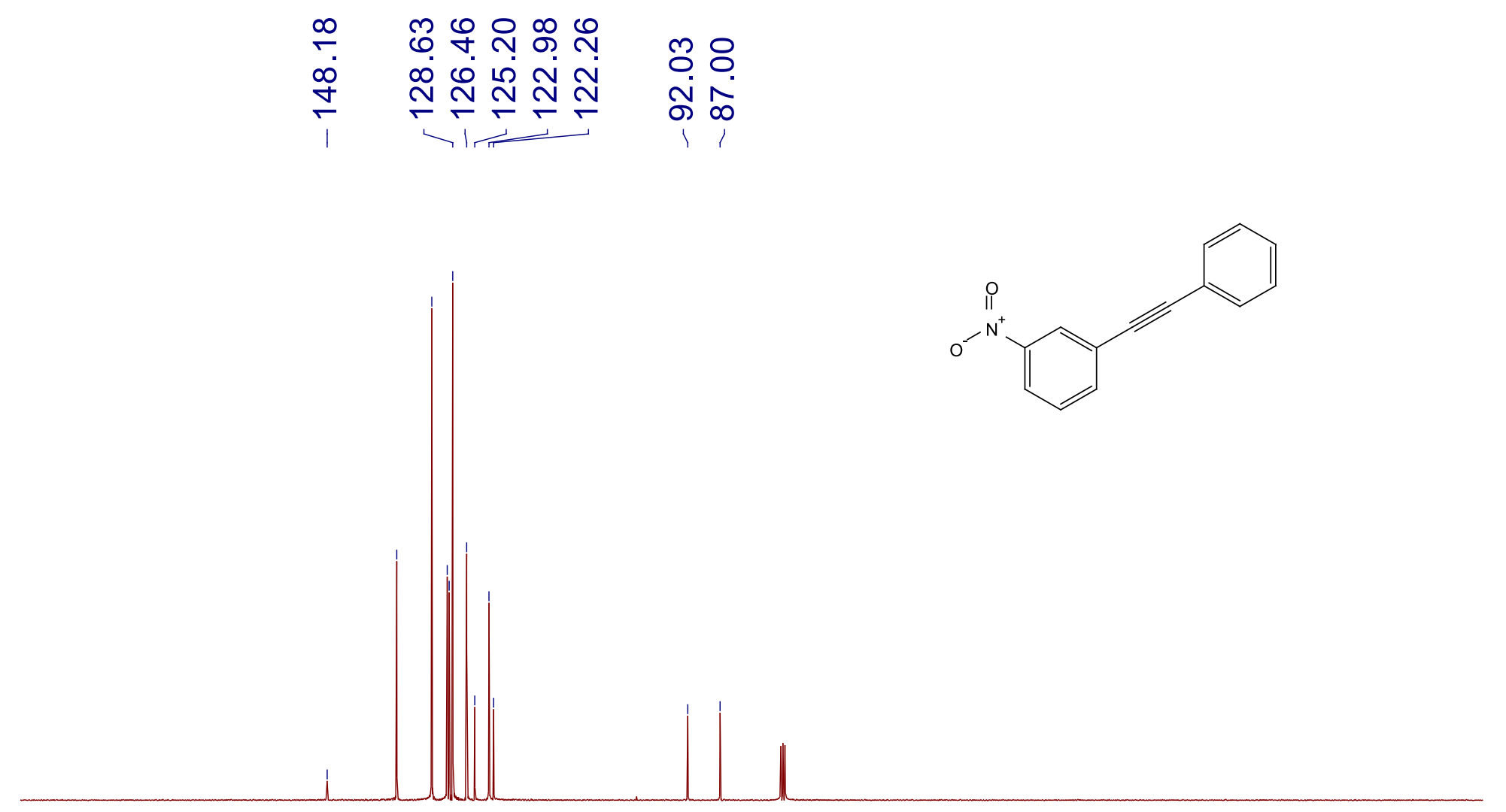

180 


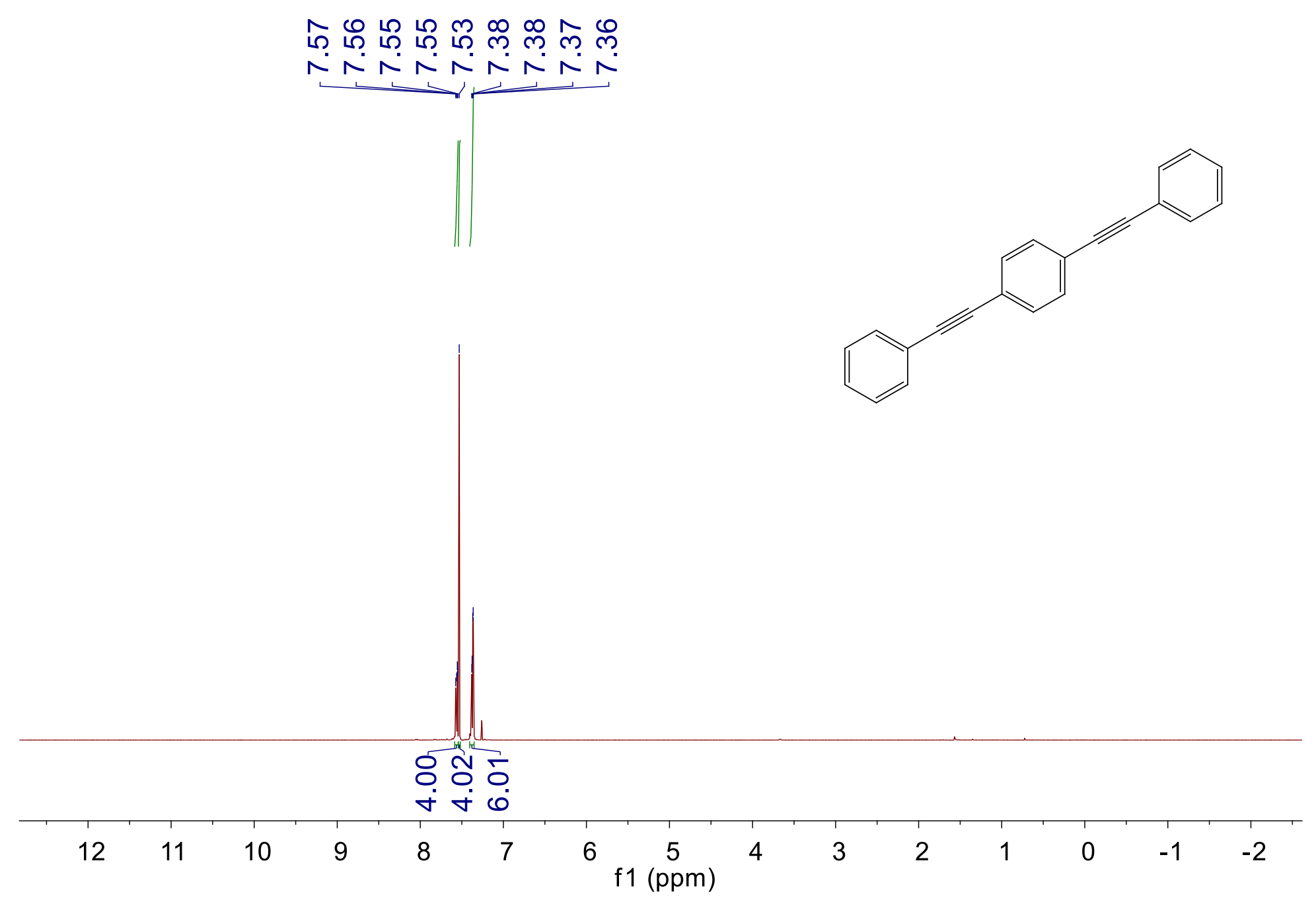




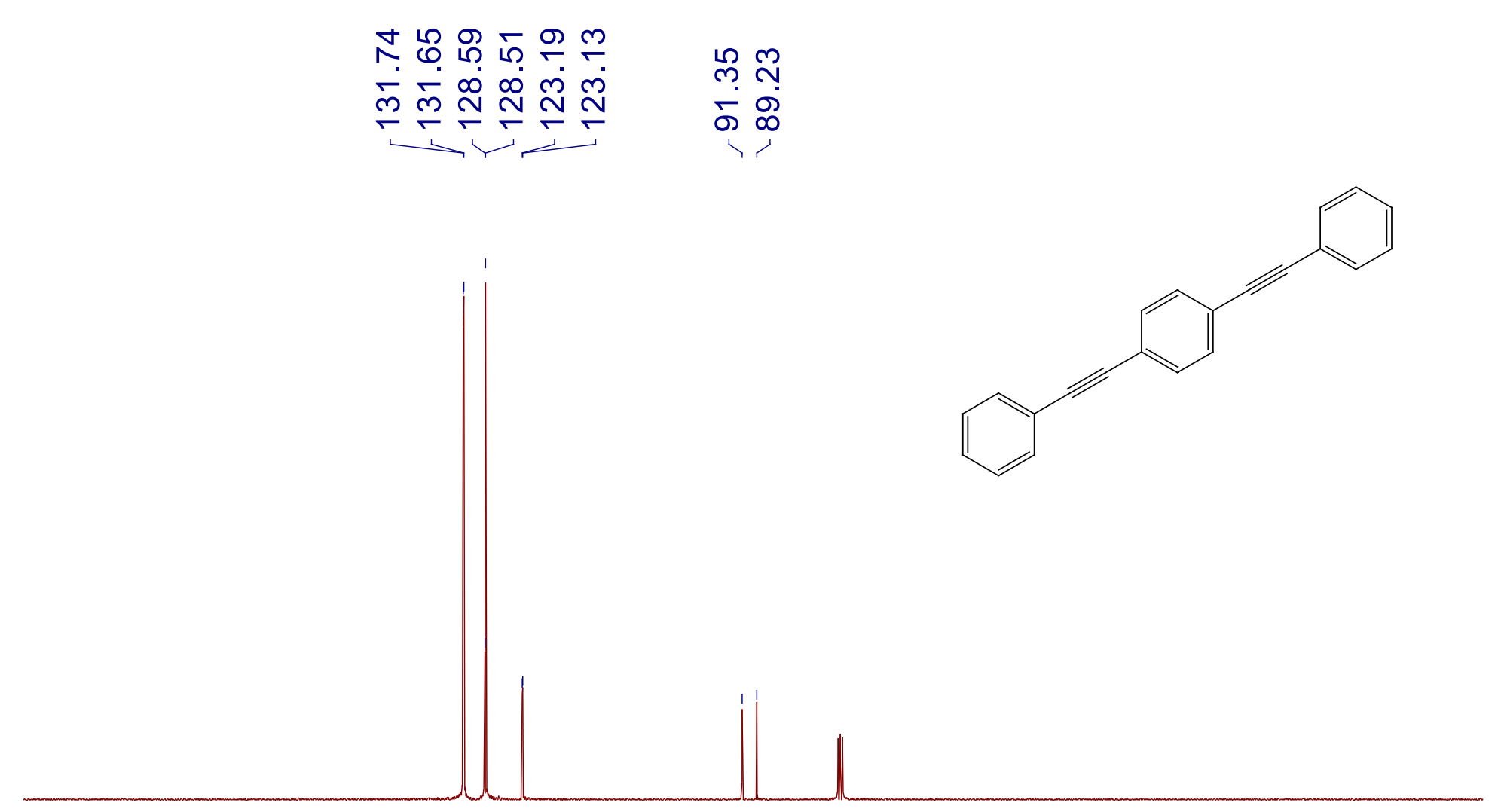

\begin{tabular}{|c|c|c|c|c|c|c|c|c|c|}
\hline 180 & 160 & 140 & 120 & 100 & $\begin{array}{l}80 \\
\text { n) }\end{array}$ & 60 & 40 & 20 & 0 \\
\hline
\end{tabular}




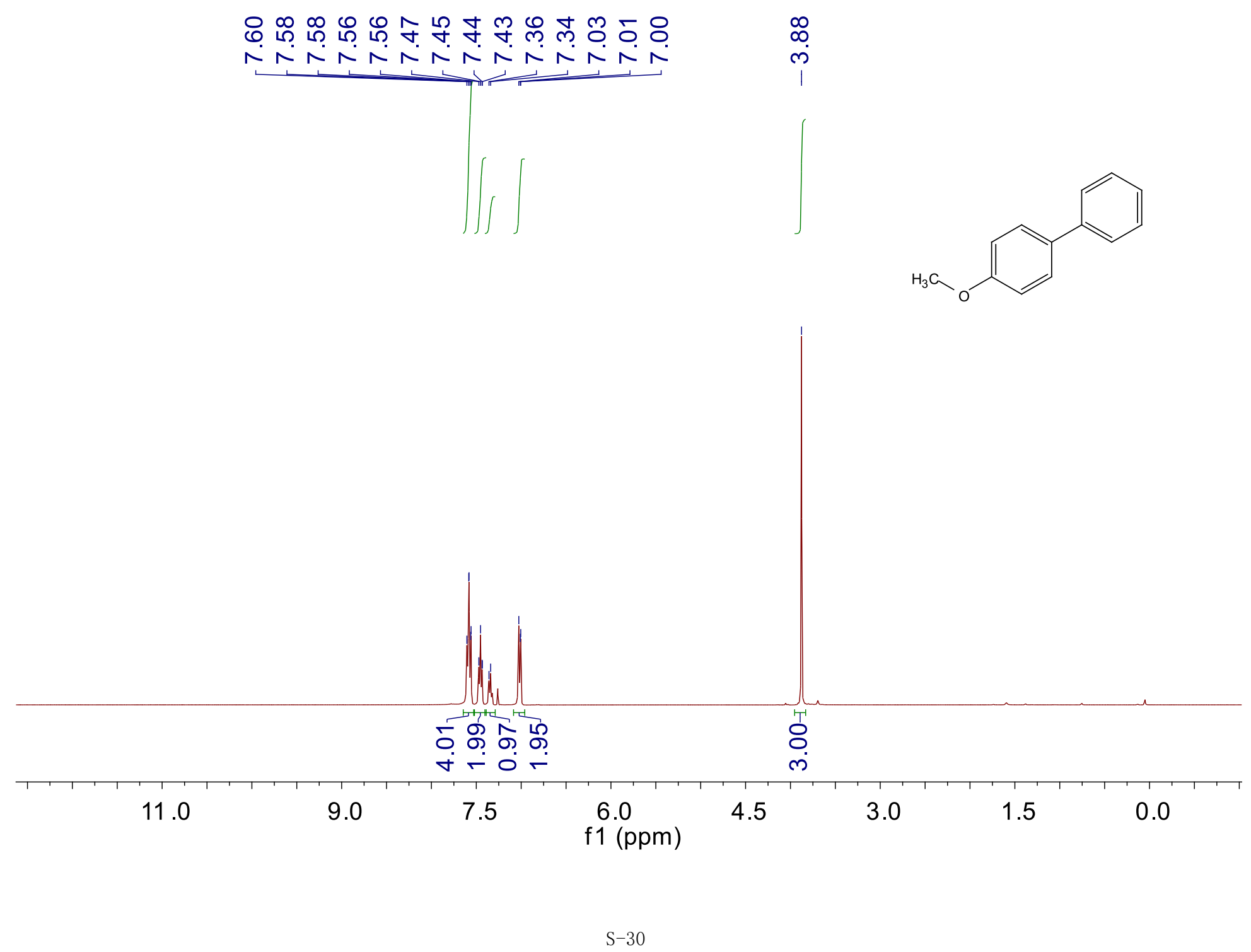




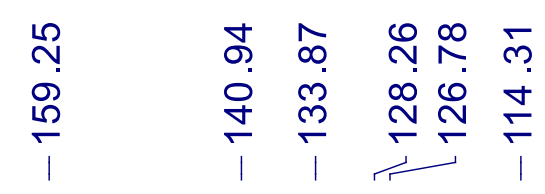

迎

นึ

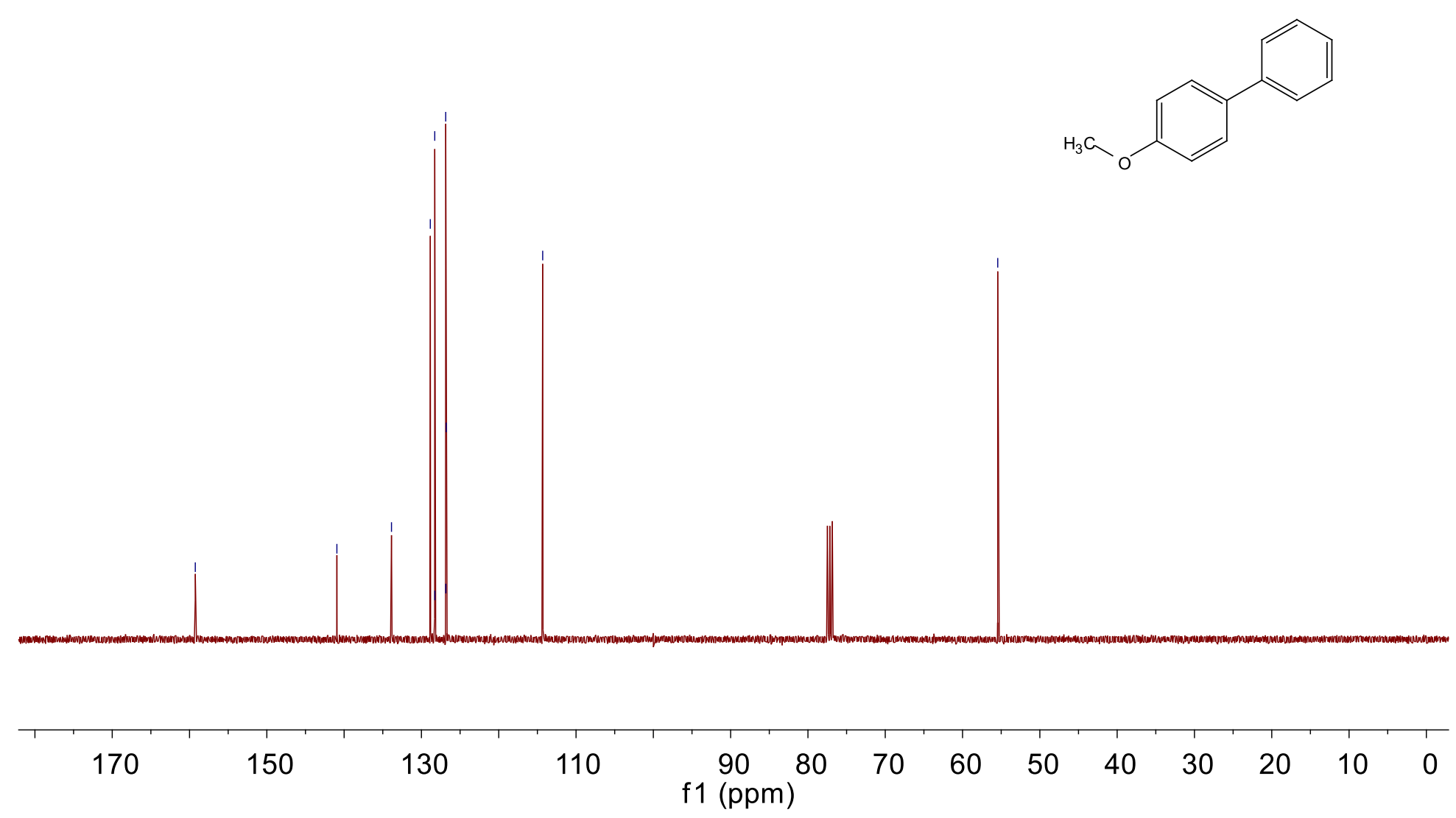



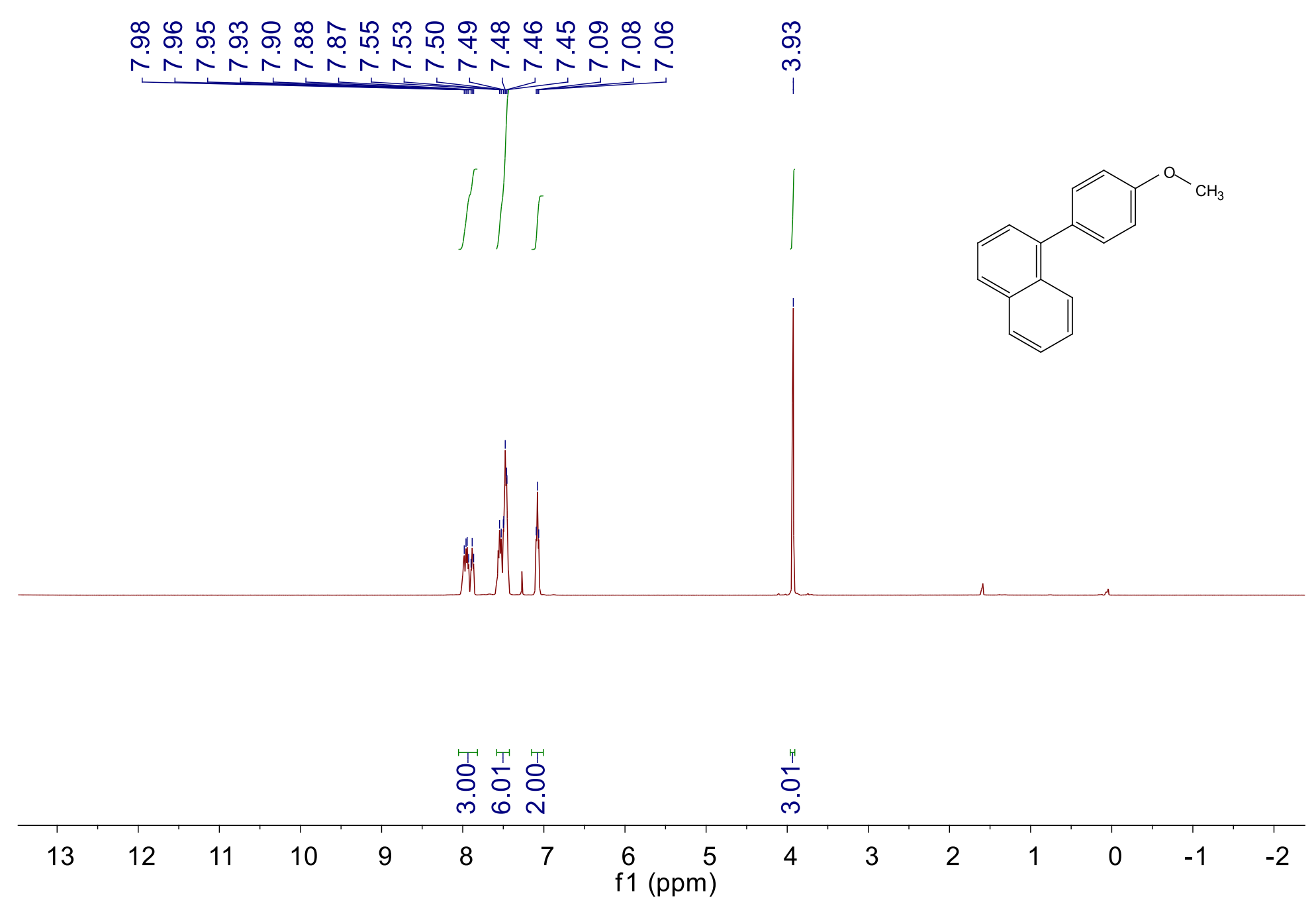


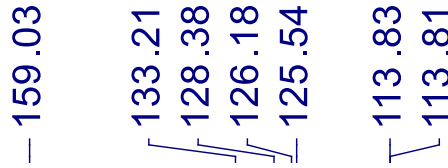

守

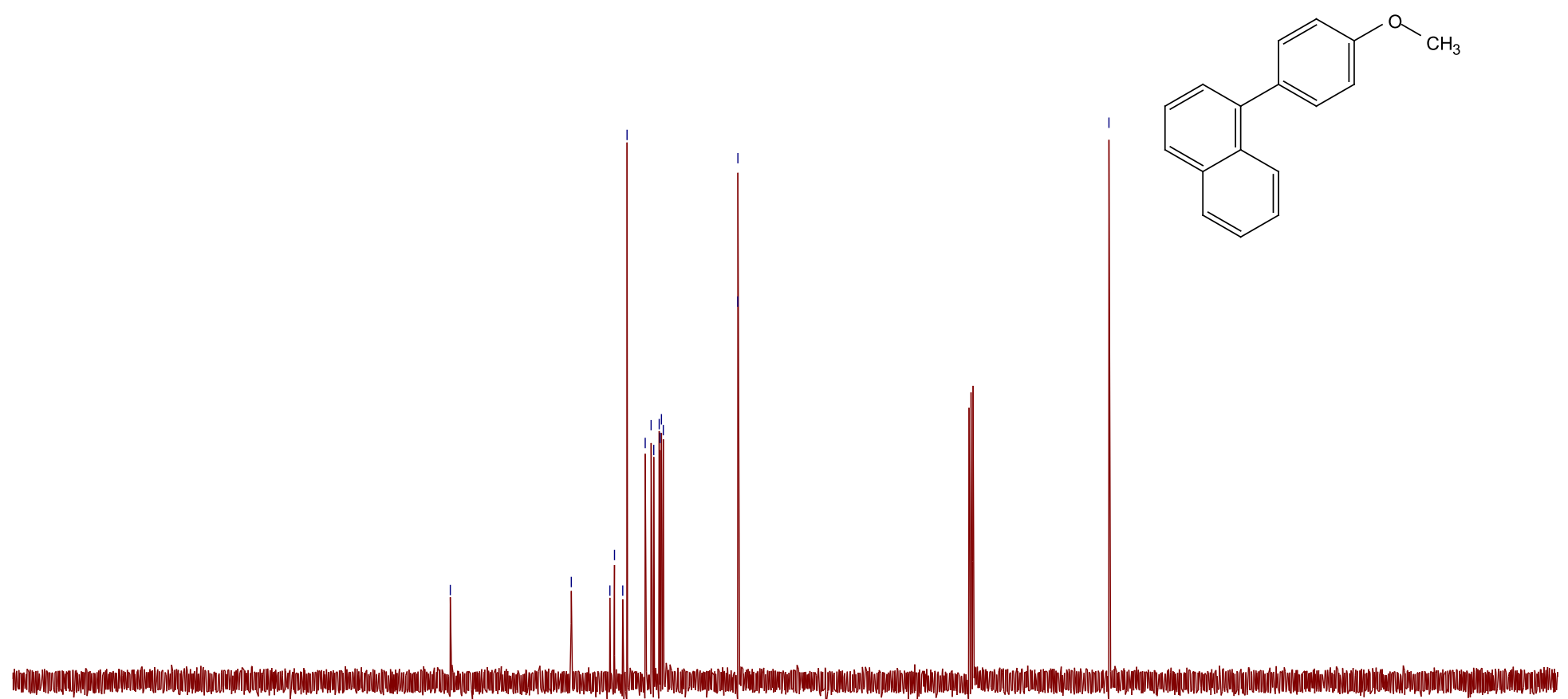

f1 (ppm)

80

60

40

20 


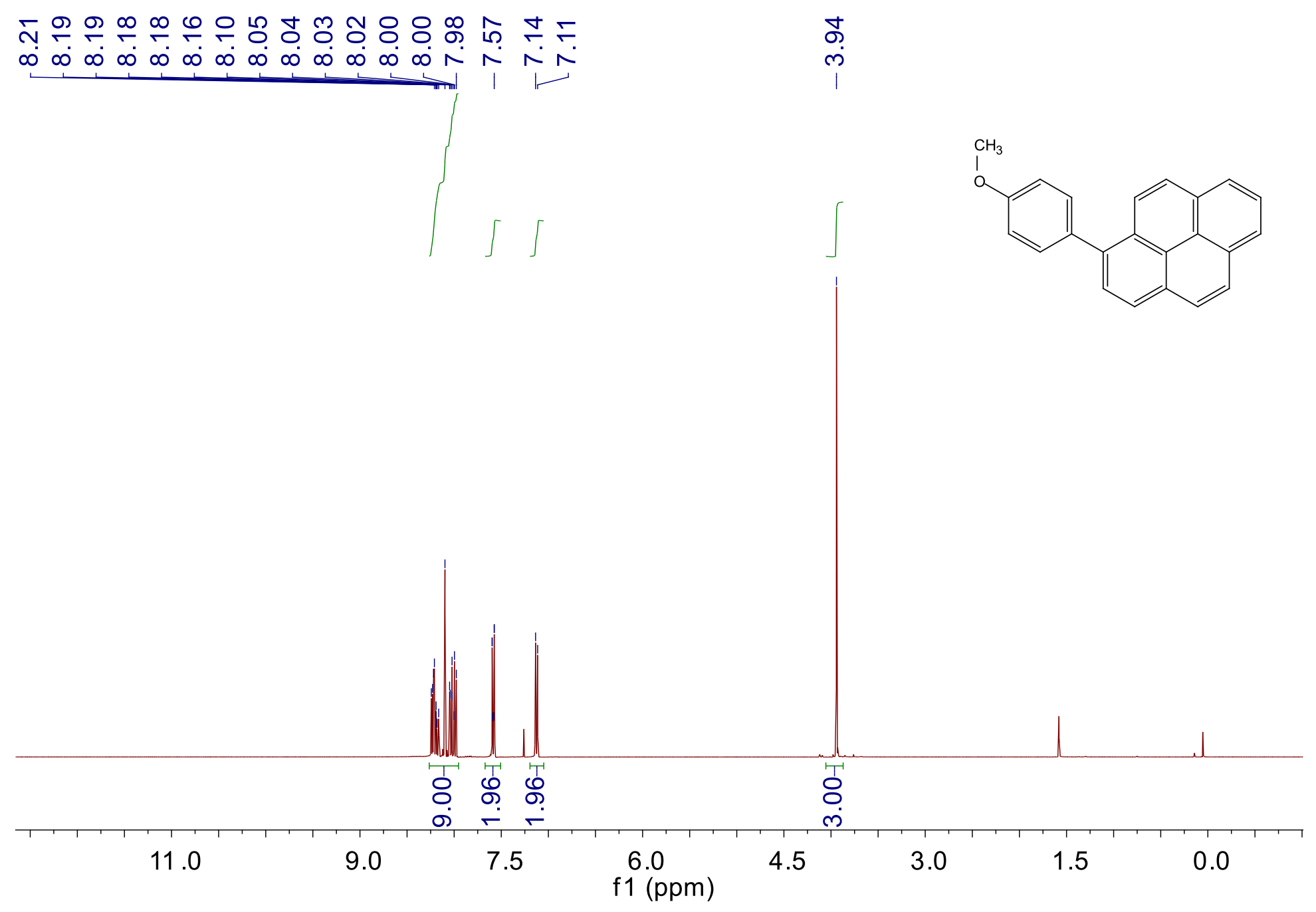



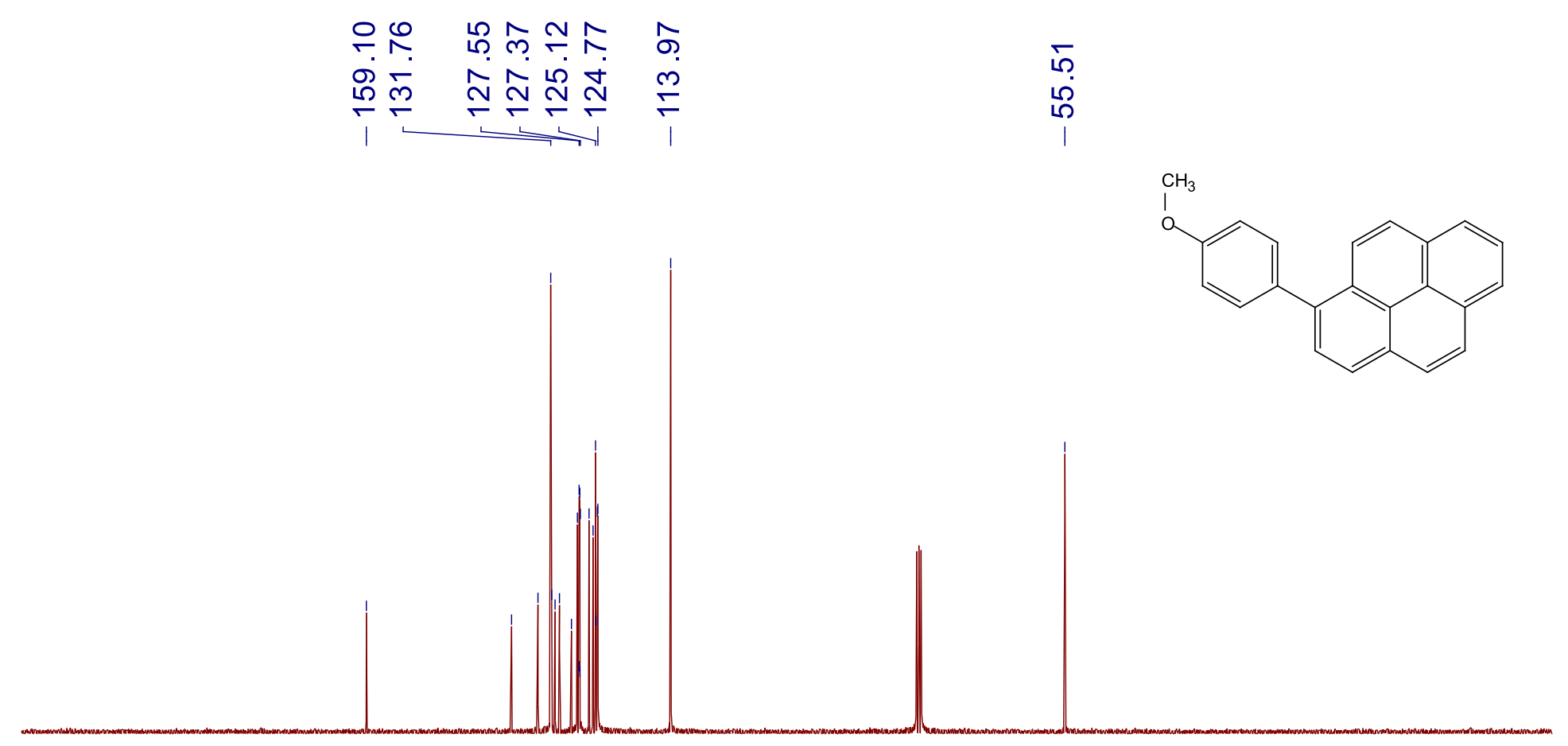


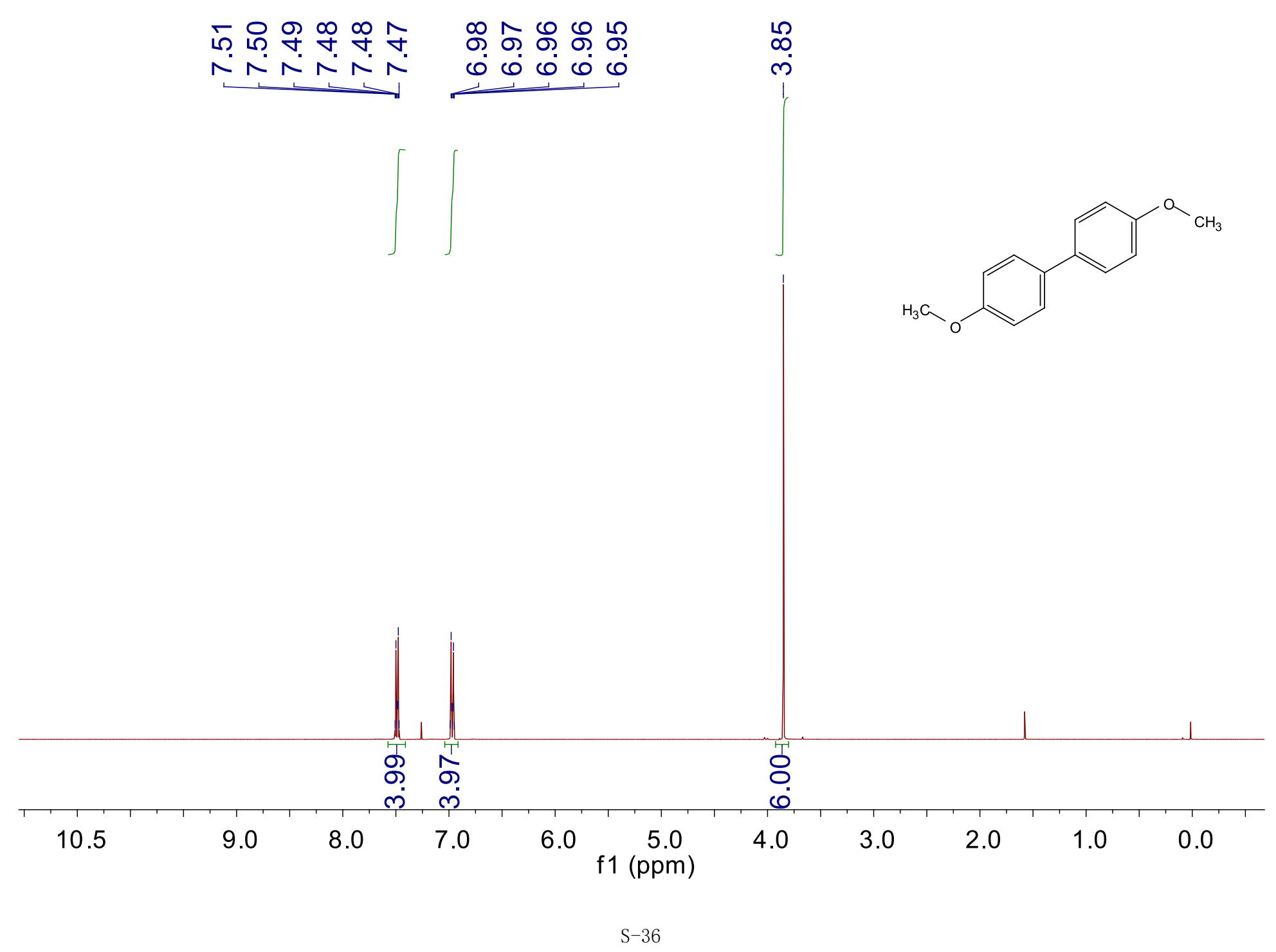




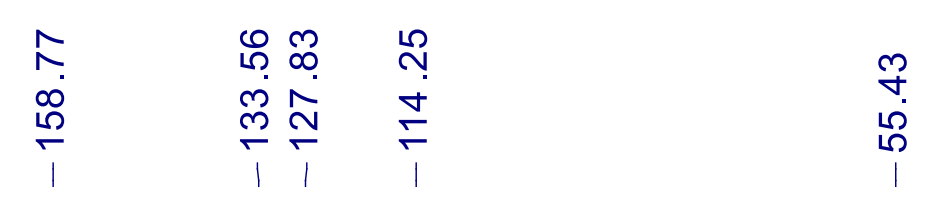

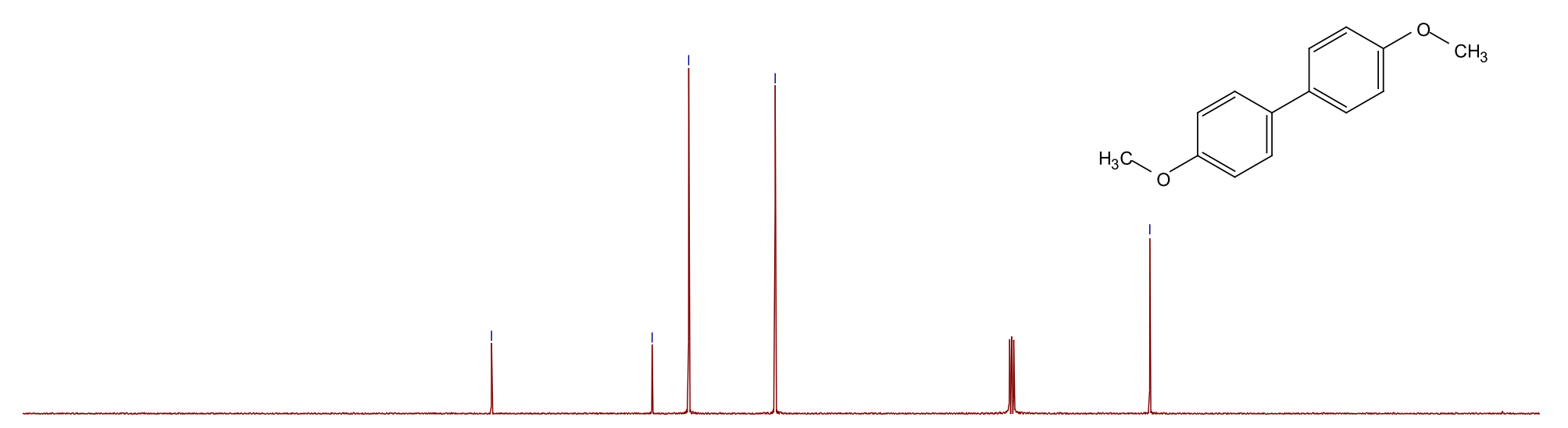

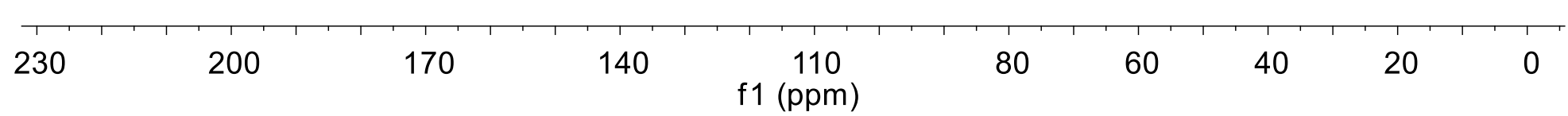

